\title{
Interference tables: a useful model for interference analysis in asynchronous multicarrier transmission
}

\author{
Yahia Medjahdi ${ }^{1 *}$, Michel Terré2${ }^{2}$, Didier Le Ruyet ${ }^{2}$ and Daniel Roviras ${ }^{2}$
}

\begin{abstract}
In this paper, we investigate the impact of timing asynchronism on the performance of multicarrier techniques in a spectrum coexistence context. Two multicarrier schemes are considered: cyclic prefix-based orthogonal frequency division multiplexing (CP-OFDM) with a rectangular pulse shape and filter bank-based multicarrier (FBMC) with physical layer for dynamic spectrum access and cognitive radio (PHYDYAS) and isotropic orthogonal transform algorithm (IOTA) waveforms. First, we present the general concept of the so-called power spectral density (PSD)-based interference tables which are commonly used for multicarrier interference characterization in spectrum sharing context. After highlighting the limits of this approach, we propose a new family of interference tables called 'instantaneous interference tables'. The proposed tables give the interference power caused by a given interfering subcarrier on a victim one, not only as a function of the spectral distance separating both subcarriers but also with respect to the timing misalignment between the subcarrier holders. In contrast to the PSD-based interference tables, the accuracy of the proposed tables has been validated through different simulation results. Furthermore, due to the better frequency localization of both PHYDYAS and IOTA waveforms, FBMC technique is demonstrated to be more robust to timing asynchronism compared to OFDM one. Such a result makes FBMC a potential candidate for the physical layer of future cognitive radio systems.
\end{abstract}

Keywords: PSD; Interference table; Time asynchronism; OFDM; FBMC; PHYDYAS; IOTA; Spectrum coexistence

\section{Introduction}

Nowadays, we witness a continuous evolution of applications for wireless communications requiring higher and higher spectral resources. In order to overcome the problem of spectrum scarcity resulting from conventional static spectrum allocation, there is a growing interest in the design and the development of cognitive radio technology [1]. The concept of cognitive radio is based on opportunistic access to the available frequency resources. It offers to future communication systems the ability to dynamically and locally adapt their operating spectrum by selecting it from a wide range of possible frequencies.

Multicarrier techniques are promising and potential candidates offering flexible access to these new spectrum opportunities [2]. Indeed, orthogonal frequency division

*Correspondence: yahia.medjahdi@uclouvain.be

1 ICTEAM, Université catholique de Louvain, Place du Levant, 2 Louvain-la-Neuve 1348, Belgium

Full list of author information is available at the end of the article multiplexing (OFDM), which is the most commonly used multicarrier technique, has been adopted in IEEE 802.22 standard for unlicensed wireless regional area network (WRAN) using cognitive communications on the unused TV bands [3]. Unfortunately, OFDM presents some weaknesses. In fact, the redundancy, caused by the insertion of the cyclic prefix mandatory part of the transmitted OFDM symbol, reduces the useful data rate. Furthermore, it has a limited frequency resolution due to the large sidelobes generated by the rectangular pulse shape frequency response. These shortcomings have stimulated the research for an alternative scheme that can overcome these problems.

In the last few years, a number of papers, e.g., [4-7] have focused on an enhanced physical layer based on the filter bank processing called filter bank-based multicarrier (FBMC) technique which can offer a number of advantages compared to CP-OFDM systems such as the 
improved spectral efficiency by not using a redundant $\mathrm{CP}$ and by having much better control of out-of-band emission, thanks to the time-frequency localized shaping pulses $[8,9]$. In the literature, we find two typical waveforms that are used in filter bank systems: the isotropic orthogonal transform algorithm (IOTA) [10] and the reference physical layer for dynamic spectrum access and cognitive radio (PHYDYAS) [11] prototype filter [12].

However, due to various factors, e.g., the propagation delays and the spatial distribution of users, timing asynchronism is considered as one of the most challenging issues in spectrum coexistence contexts. Indeed, the timing asynchronism between coexisting systems can harmfully affect the performance by causing the so-called asynchronous interference. Consequently, it is relevant to evaluate the impact of this asynchronism on the system performance.

Interference modeling is an important problem, with numerous applications to the analysis and design of multiuser communication systems, as well as the development of interference mitigation techniques. This problem has been intensively investigated in the literature through the most common approach using the power spectral density (PSD) $[8,9,13,14]$. This model is based on the out-of-band radiation which is determined by the PSD model of multicarrier signals. However, this model does not always give accurate results. For example, in multiuser CP-OFDM when the timing offset does not exceed the cyclic prefix duration, the interference comes only from the same subchannel and the other subchannels do not contribute to this interference. Unfortunately, in this case, the PSD modeling still shows that the other carriers contribute in the resulting interference.

It is worth mentioning that there are already several works in the literature showing the FBMC robustness to time asynchronism. In [15-17], the authors demonstrate that in an asynchronous multi-user scenario, FBMC systems are more robust than OFDM systems to time and frequency misalignments among the users. Moreover, the sensitivity of the different FBMC waveforms is investigated in [18]. However, we want to indicate that the interference modeling proposed in this paper is general and can be used for any multicarrier scheme. Moreover, this model is a more efficient alternative to overcome the limitations of the PSD-based modeling which is commonly used in the analysis of interference in coexistence contexts.

In this paper, the impact of timing asynchronism on the performance of OFDM and FBMC systems is addressed. As a matter of fact, we would like to:

- Properly estimate the interference part introduced by the timing asynchronism for OFDM systems.
- Propose an extension of this model to the FBMC case, as it has, to our knowledge, never been considered in the literature.

- Extend the interference analysis to the case of frequency selective environments.

- Evaluate the accuracy of the proposed model through different simulation results.

The rest of this paper is organized as follows. Section 2 presents the general notion of the so-called interference tables, where we give the PSD-based interference tables of CP-OFDM and FBMC considering PHYDYAS and IOTA prototype filters. In Section 3, we derive the OFDM/FBMC instantaneous interference tables taking into account the timing asynchronism in addition to the spectral distance between the interfering user and the victim one. Next, we extend the interference analysis to the case of frequency selective environments in Section 4. The accuracy of the proposed interference modeling is then investigated in Section 5. We finally conclude the paper in Section 6.

\subsection{Definitions and notations}

In this paper, we are calculating interference weights that can be used in the estimation of interference in coexistence contexts. Since these weights are computed as a function of two parameters, the timing offset and the spectral distance between the coexisting systems, we then obtain a 2D table of these weights for each multicarrier scheme. Thus, we find it appropriate to call them interference tables'. For simplicity sake, we also use this definition in the PSD-based approach.

\section{The general concept of interference tables}

Let us consider two asynchronous systems (A) and (B) that coexist in the same geographical area. We assume that both systems share a given frequency band where $\mathcal{F}_{A}$ and $\mathcal{F}_{B}$ are the frequency sub-bands occupied by systems (A) and (B), respectively.

Due to the non-orthogonality between their respective transmit signals, some amount of the power is spilled from a system to the other. In order to analyze this interaction, the PSD is generally used to evaluate the mutual interference between both systems $[9,13]$. According to [13], the normalized mutual interference between the co-located systems is defined as

$$
I(l)=\int_{(l-1 / 2) \Delta f}^{(l+1 / 2) \Delta f} \Phi(f) d f,
$$

where:

- $l$ is the spectral distance between the two interacting subcarriers. 
- $\Delta f$ is the subcarrier spacing.

- $\Phi(f)$ is the PSD which depends on the considered multicarrier technique.

In the case of CP-OFDM system, the normalized PSD is given in [13] by

$$
\Phi_{\text {OFDM }}(f)=T_{\text {OFDM }}\left(\frac{\sin \left(\pi f T_{\text {OFDM }}\right)}{\pi f T_{\text {OFDM }}}\right)^{2},
$$

where $T_{\mathrm{OFDM}}$ is the OFDM symbol duration given by the sum of the useful symbol duration $T$ and the $\mathrm{CP}$ duration $\Delta$.

The red curve of Figure 1 depicts the normalized OFDM PSD. The mutual interference power when $l=1$ corresponds to the gray-colored area.

The PSD of FBMC systems has been computed in [9], for two waveforms: IOTA and PHYDYAS. The respective expressions are as follows:

$$
\begin{aligned}
& \Phi_{\text {IOTA }}(f)=\left(g_{1, \sqrt{2} / 2, \sqrt{2} / 2}(t)\right)^{2} . \\
& \Phi_{\text {PHYDYAS }}(f)=(G(f))^{2} .
\end{aligned}
$$

Here, $g_{1, \sqrt{2} / 2, \sqrt{2} / 2}(t)$ is the impulse response of the IOTA filter. The derivation of the latter is detailed in [10]. $G(f)$ is the square root of the PHYDYAS filter frequency response which is given by

$$
G(f)=\sum_{k=-(K-1)}^{k=(K-1)} G_{k} \frac{\sin \left(\pi\left(f-\frac{k}{N K}\right) N K\right)}{N K \sin \left(\pi\left(f-\frac{k}{N K}\right)\right)},
$$

where the coefficients $G_{k}$ are given by $[12,19]$

$$
\begin{aligned}
& G_{0}=1, G_{1}=0.971960, G_{2}=1 / \sqrt{2}, \\
& G_{3}=\sqrt{1-G_{1}^{2}}, G_{k}=0 ; 4<k<L-1 .
\end{aligned}
$$

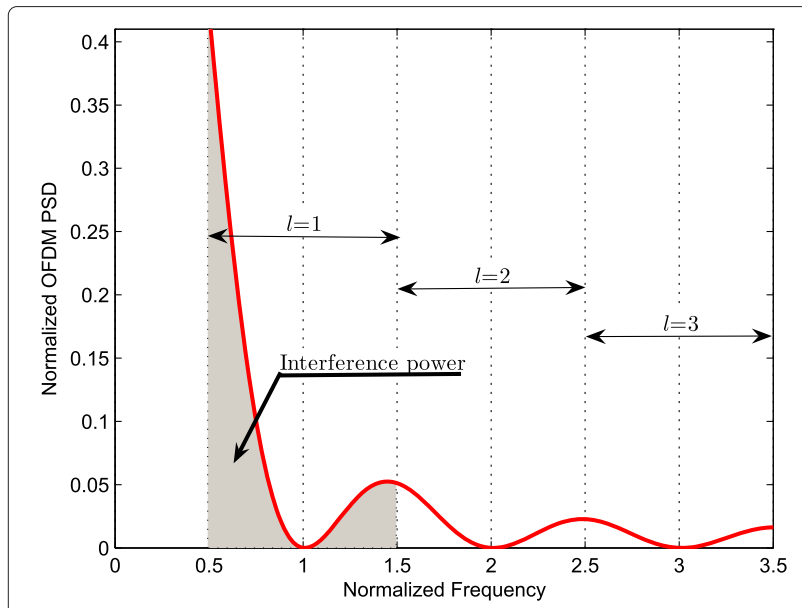

Figure 1 PSD of a single OFDM modulated carrier.
Based on Equation 1, we can construct a table of mutual interference as a function of the spectral distance $l$. The PSD-based interference tables for CP-OFDM are given in Table 1 for different values of CP duration: $\Delta=0, T / 8$ and $T / 4$. Moreover, we give in Table 2 the PSD-based interference tables for FBMC considering PHYDYAS and IOTA waveforms [9].

It is worth to point out that these tables are symmetrical as shown in Figure 2.

In Figure 3, we illustrate how to compute the interference caused by the different subcarriers of $\mathcal{F}_{A}$ to a given subcarrier $m$ of system (B). The total interference is the sum of the contribution of each interfering subcarrier $m^{\prime}$, and can thus be written as

$$
I_{\text {tot }}=\sum_{m^{\prime} \in \mathcal{F}_{A}} P\left(m^{\prime}\right) I\left(\left|m^{\prime}-m\right|\right)
$$

where $P\left(m^{\prime}\right)$ is the transmitted power on the $m^{\prime}$-th interfering subcarrier and $I\left(m^{\prime}-m\right)$ is the PSD-based interference weight computed in Tables 1 and 2 .

Various analysis have been developed based on this interference estimation, e.g., SINR, spectral efficiency analysis in $[9,20]$, and resource allocation algorithms $[14,20]$.

According to (1), one can see that the interference remains the same for any timing misalignment between the transmitted signals of both systems since the signals are considered to be non-orthogonal. However, in CP-OFDM systems, the orthogonality between the different transmit signals is maintained as long as the timing misalignment does not exceed the cyclic prefix dura-

\begin{tabular}{|c|c|c|c|}
\hline$I$ & $\begin{array}{c}\Delta=0 \\
{[\mathrm{~dB}]}\end{array}$ & $\begin{array}{c}\Delta=T / 8 \\
{[\mathrm{~dB}]}\end{array}$ & $\begin{array}{c}\Delta=T / 4 \\
{[\mathrm{~dB}]}\end{array}$ \\
\hline 0 & -01.11 & -00.87 & -00.70 \\
\hline 1 & -11.04 & -12.00 & -13.08 \\
\hline 2 & -18.52 & -19.72 & -20.72 \\
\hline 3 & -22.30 & -23.55 & -23.16 \\
\hline 4 & -24.88 & -25.98 & -25.14 \\
\hline 5 & -26.86 & -27.62 & -27.97 \\
\hline 6 & -28.46 & -28.83 & -30.34 \\
\hline 7 & -29.82 & -29.92 & -30.72 \\
\hline 8 & -31.00 & -31.05 & -31.24 \\
\hline
\end{tabular}
tion. This example highlights the overestimation of the asynchronous interference term. In fact, the real asynchronous interference is always a function of the timing offset between the considered asynchronous systems that is not taken into account in the PSD-based interference tables generation. 
Table 2 FBMC mutual interference tables based on the PSD for PHYDYAS and IOTA waveforms, respectively

\begin{tabular}{lcc}
\hline $\boldsymbol{I}$ & $\begin{array}{c}\text { PHYDYAS } \\
{[\mathbf{d B}]}\end{array}$ & $\begin{array}{c}\text { IOTA } \\
{[\mathbf{d B}]}\end{array}$ \\
\hline 0 & -00.58 & -00.70 \\
1 & -11.95 & -09.27 \\
2 & -65.02 & -35.56 \\
3 & -80.30 & -42.55 \\
4 & -89.22 & -65.17 \\
5 & -95.68 & -71.72 \\
6 & -100.80 & -87.31 \\
7 & -105.08 & -90.53 \\
8 & -108.80 & -91.45 \\
\hline
\end{tabular}

In the next section, we propose new interference tables that model the correlation between the interfering subcarrier and the victim one considering the timing offset between them in addition to the different parameters already considered by the PSD-based interference tables.

\section{Instantaneous OFDM/FBMC interference tables}

To take into account the detrimental effects of interference caused by the imperfect synchronization in multicarrier techniques, we consider the system model depicted in Figure 4. We refer, here, to a receiver which suffers from the interference coming from an asynchronous transmitter. This receiver is assumed to be perfectly synchronized with its corresponding transmitter. Moreover, the timing offset $\tau$ and the phase offset $\varphi$ are assumed to be uniform random variables that are distributed on $\tau \in[0, T]$ and $\varphi \in[0,2 \pi]$, respectively. In the following analysis, we will be interested in the impact of the interfering signal $s(t-\tau)$ on the reference receiver.

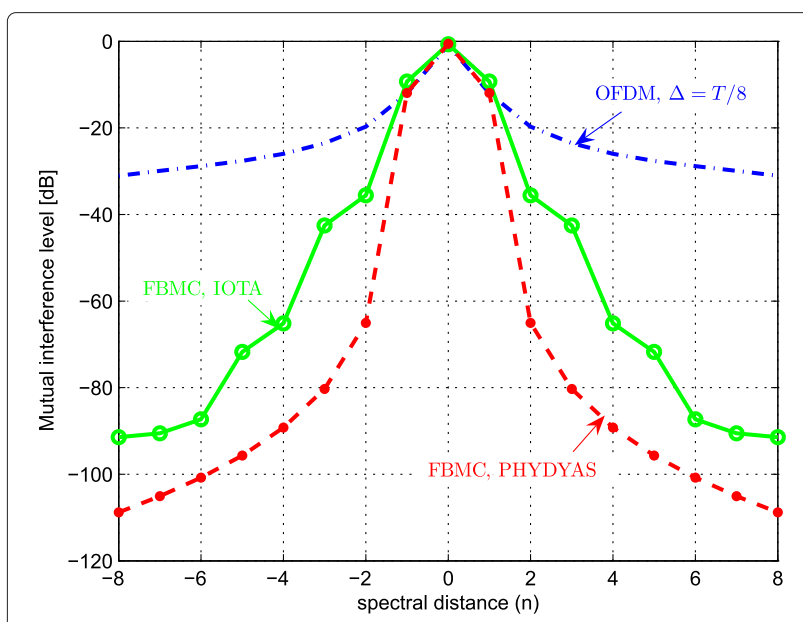

Figure 2 PSD-based interference tables of OFDM and FBMC.

\subsection{CP-OFDM case}

Consider the following asynchronous signal coming from the interferer on the $m$-th subcarrier

$$
\begin{aligned}
s_{m}(t-\tau, \varphi)= & \sum_{n=-\infty}^{n=+\infty} x_{m, n} f_{T}(t-n(T+\Delta)-\tau) \\
& \times e^{j\left[\frac{2 \pi}{T} m(t-n(T+\Delta)-\tau)+\varphi\right]}
\end{aligned}
$$

where

- $x_{m, n}$ are the complex data symbols transmitted by the interferer.

- $T$ and $\Delta$ are the useful OFDM symbol duration and the CP duration, respectively.

- The timing offset and the phase offset between the reference receiver and the interferer are respectively denoted by $\tau$ and $\varphi$.

Here, $f_{T}(t)$ and $f_{R}(t)$ are, respectively, the transmit and the receiver pulse shapes,

$$
\begin{aligned}
f_{T}(t) & = \begin{cases}\frac{1}{\sqrt{T}} & t \in[0, T+\Delta] \\
0 & \text { elsewhere }\end{cases} \\
f_{R}(t) & = \begin{cases}\frac{1}{\sqrt{T}} & t \in[\Delta, T+\Delta] \\
0 & \text { elsewhere }\end{cases}
\end{aligned}
$$

The $m_{0}$-th output of the receiver filter on the $n_{0}$-th signaling interval coming from $s_{m}(t-\tau, \varphi)$, will be

$$
\begin{aligned}
y_{m_{0}, n_{0}}(\tau, \varphi)= & \left\langle s_{m}(t-\tau, \varphi), f_{R}\left(t-n_{0}(T+\Delta)\right)\right. \\
& \left.\times e^{j \frac{2 \pi}{T} m_{0}\left(t-n_{0}(T+\Delta)\right)}\right\rangle \\
= & \int_{-\infty}^{+\infty} s_{m}(t-\tau, \varphi) f_{R}\left(t-n_{0}(T+\Delta)\right) \\
& \times e^{-j \frac{2 \pi}{T} m_{0}\left(t-n_{0}(T+\Delta)\right)} d t \\
= & \sum_{n=-\infty}^{+\infty} x_{m, n} e^{-j\left[\frac{2 \pi}{T} m \tau-\varphi\right]} \int_{-\infty}^{+\infty} f_{T}(t-n(T+\Delta)-\tau) \\
& \times f_{R}\left(t-n_{0}(T+\Delta)\right) e^{j \frac{2 \pi}{T} m(t-n(T+\Delta))} \\
& \times e^{-j \frac{2 \pi}{T} m_{0}\left(t-n_{0}(T+\Delta)\right)} d t
\end{aligned}
$$

where, $\langle.,$.$\rangle stands for the inner product.$ 


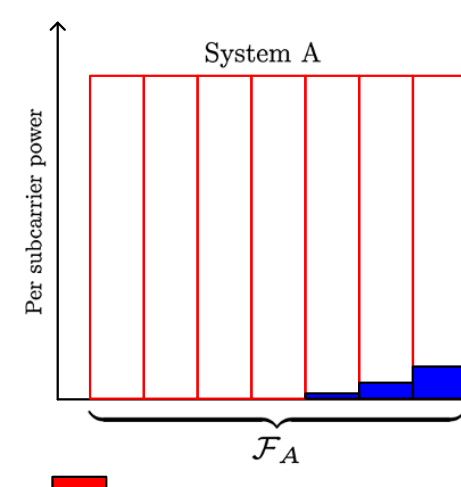

interference power from (A) to (B)
System B

$\sum_{m^{\prime} \in \mathcal{F}_{A}} P\left(m^{\prime}\right) I\left(\left|m^{\prime}-m\right|\right)$

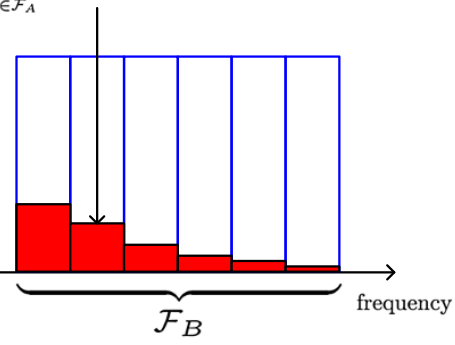

interference power from (B) to (A)

Figure 3 Illustration of how to compute the interference caused by a set of subcarriers.

In the general case, we see that the product $f_{T}(t-n(T+$ $\Delta)-\tau) f_{R}\left(t-n_{0}(T+\Delta)\right)$ and the choice of $\tau$ determine the limits of the integral appearing in (9), we have then two cases to analyze

\subsubsection{Case 1: $(0<\tau<\Delta)$}

In this case, the positions of the receiver window and the interferer window are depicted in Figure 5 and the signal $y_{m_{0}, n_{0}}(\tau)$ becomes

$$
\begin{aligned}
& y_{m_{0}, n_{0}}(\tau, \varphi)= x_{m, n_{0}} e^{-j\left[\frac{2 \pi}{T} m \tau-\varphi\right]} \\
& \int_{n_{0}(T+\Delta)+\Delta}^{\left(n_{0}+1\right)(T+\Delta)} \frac{1}{T} e^{j \frac{2 \pi}{T}\left(m-m_{0}\right)\left(t-n_{0}(T+\Delta)\right)} d t \\
&= \begin{cases}x_{m_{0}, n_{0}} e^{-j\left[\frac{2 \pi}{T} m_{0} \tau-\varphi\right]} & m=m_{0} \\
0 & \text { otherwise }\end{cases}
\end{aligned}
$$

Here, the timing offset $\tau$ is absorbed by the cyclic prefix $\Delta$. The interference will only occur on the same subcarrier $m=m_{0}$, the other subcarriers are free of interference due to the orthogonality between them.

\subsubsection{Case 2: $(\Delta<\tau<T+\Delta)$}

As illustrated in Figure 6, the product $f_{T}(t-n(T+\Delta)-$ $\tau) f_{R}\left(t-n_{0}(T+\Delta)\right)$ is nonzero when $n=n_{0}-1$ and $n=n_{0}$, simultaneously. Consequently, the signal $y_{m_{0}, n_{0}}(\tau)$ can be written as follows:

$$
\begin{aligned}
& y_{m_{0}, n_{0}}(\tau, \varphi)=e^{-j\left[\frac{2 \pi}{T} m \tau-\varphi\right]}\left\{x_{m, n_{0}-1} \int_{n_{0}(T+\Delta)+\Delta}^{n_{0}(T+\Delta)+\tau} \frac{1}{T}\right. \\
& +e^{j \frac{2 \pi}{T} m\left(t-\left(n_{0}-1\right)(T+\Delta)\right)} e^{-j \frac{2 \pi}{T} m_{0}\left(t-n_{0}(T+\Delta)\right)} d t x_{m, n_{0}} \\
& \left.\times \int_{n_{0}(T+\Delta)+\tau}^{\left(n_{0}+1\right)(T+\Delta)} \frac{1}{T} e^{j \frac{2 \pi}{T}\left(m-m_{0}\right)\left(t-n_{0}(T+\Delta)\right)} d t\right\}
\end{aligned}
$$

$$
=e^{-j\left[\frac{2 \pi}{T} m \tau-\varphi\right]}\left\{\frac{x_{m, n_{0}-1}}{T} e^{-j \frac{2 \pi}{T} m(T+\Delta)} \int_{n_{0}(T+\Delta)+\Delta}^{n_{0}(T+\Delta)+\tau}\right.
$$

$$
\begin{aligned}
& \times e^{j \frac{2 \pi}{T}\left(m-m_{0}\right)\left(t-n_{0}(T+\Delta)\right)} d t \frac{x_{m, n_{0}}}{T} \\
& \left.+\int_{n_{0}(T+\Delta)+\tau}^{\left(n_{0}+1\right)(T+\Delta)} e^{j \frac{2 \pi}{T}\left(m-m_{0}\right)\left(t-n_{0}(T+\Delta)\right)} d t\right\} .
\end{aligned}
$$

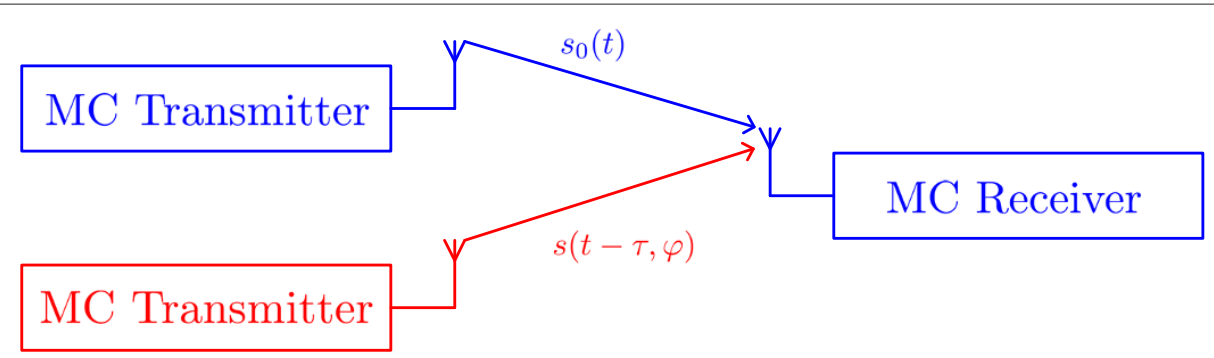

Figure 4 Asynchronous interference in multicarrier systems. 


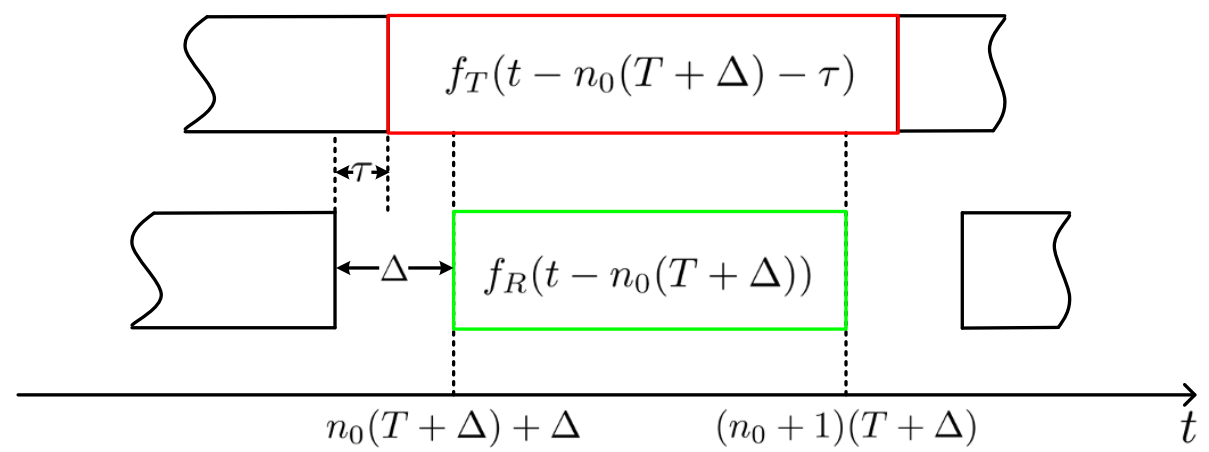

Figure 5 Respective positions of transmit and receiver pulses when $\tau \in[0, \Delta]$.

When $m \neq m_{0}$ equation (11) is reduced, upon the change of variables $t=t^{\prime}-n_{0}(T+\Delta)$, to

$$
\begin{aligned}
y_{m_{0}, n_{0}}(\tau, \varphi)= & e^{-j\left[\frac{2 \pi}{T} m \tau-\varphi\right]}\left\{\frac{x_{m, n_{0}-1}}{T} e^{-j \frac{2 \pi}{T} m(T+\Delta)} \int_{\Delta}^{\tau}\right. \\
& \left.\times e^{j \frac{2 \pi}{T}\left(m-m_{0}\right) t} d t+\frac{x_{m, n_{0}}}{T} \int_{\tau}^{(T+\Delta)} e^{j \frac{2 \pi}{T}\left(m-m_{0}\right) t} d t\right\} \\
= & e^{-j\left[\frac{2 \pi}{T} m \tau-\varphi\right]}\left\{\frac{x_{m, n_{0}-1}}{j 2 \pi\left(m-m_{0}\right)} e^{-j \frac{2 \pi}{T} m(T+\Delta)}\right. \\
& \times\left[e^{j \frac{2 \pi}{T}\left(m-m_{0}\right) \tau}-e^{j \frac{2 \pi}{T}\left(m-m_{0}\right) \Delta}\right]+\frac{x_{m, n_{0}}}{j 2 \pi\left(m-m_{0}\right)} \\
& \left.\times\left[e^{j \frac{2 \pi}{T}\left(m-m_{0}\right)(T+\Delta)}-e^{j \frac{2 \pi}{T}\left(m-m_{0}\right) \tau}\right]\right\} .
\end{aligned}
$$

Using some trigonometric transformations, the signal $y_{m_{0}, n_{0}}(\tau)$ can be written in the following form:

$$
\begin{aligned}
y_{m_{0}, n_{0}}(\tau, \varphi)= & e^{-j\left[\frac{2 \pi}{T} m \tau-\varphi\right]}\left\{\frac{x_{m, n_{0}-1}}{\pi\left(m-m_{0}\right)} e^{-j \frac{2 \pi}{T} m(T+\Delta)}\right. \\
& \times e^{j \frac{\pi}{T}\left(m-m_{0}\right)(\tau+\Delta)} \sin \left[\pi\left(m-m_{0}\right)(\tau-\Delta) / T\right] \\
& +\frac{x_{m, n_{0}}}{\pi\left(m-m_{0}\right)} e^{j \frac{\pi}{T}\left(m-m_{0}\right)(T+\Delta+\tau)} \\
& \left.\times \sin \left[\pi\left(m-m_{0}\right)(T+\Delta-\tau) / T\right]\right\} .
\end{aligned}
$$

when $m=m_{0}$, the signal $y_{m_{0}, n_{0}}(\tau)$ is given by

$$
\begin{aligned}
y_{m_{0}, n_{0}}(\tau, \varphi)= & e^{-j\left[\frac{2 \pi}{T} m_{0} \tau-\varphi\right]}\left\{x_{m_{0}, n_{0}-1} e^{-j \frac{2 \pi}{T} m_{0}(T+\Delta)} \frac{\tau-\Delta}{T}\right. \\
& \left.+x_{m_{0}, n_{0}} \frac{T+\Delta-\tau}{T}\right\} .
\end{aligned}
$$

Accordingly, when the timing offset $\tau$ is larger than the cyclic prefix duration $\Delta$, the orthogonality between subcarriers is damaged. Thus, the interference is caused by all subcarriers.

As the communication symbols $x_{m, n}$ are zero mean uncorrelated variables, the corresponding interference power is the sum of the interference power coming respectively from two successive data symbols $\left(x_{m, n-1}\right.$,

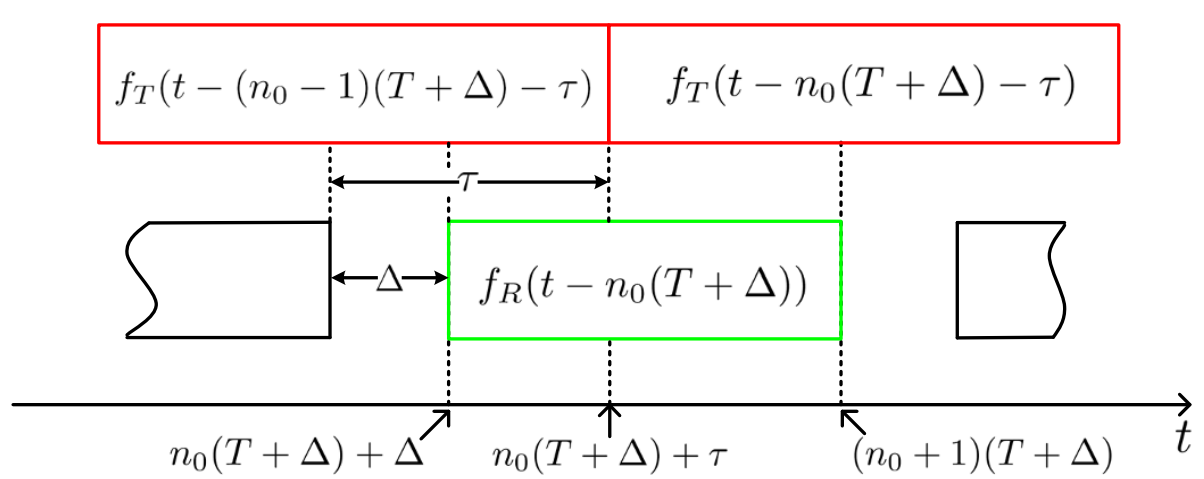

Figure 6 Respective positions of transmit and receiver pulses when $\tau \in[\Delta, T+\Delta]$. 
$\left.x_{m, n}\right)$. Without loss of generality, we assume that $\mathbb{E}\left[\left|x_{m, n}\right|^{2}\right]=1$, we can define the instantaneous interference tables by the following expression:

$$
\begin{aligned}
& I(\tau, l)=\mathbb{E}_{x}\left[\left|y_{m_{0}, n_{0}}(\tau, \varphi)\right|^{2}\right] \\
& = \begin{cases}\delta(l) & \tau \in[0, \Delta] \\
{\left[(T+\Delta-\tau)^{2}+(\tau-\Delta)^{2}\right] / T^{2}} & \tau \in[\Delta, T+\Delta], l=0 \\
\left|\frac{\sin (\pi l(T+\Delta-\tau) / T)}{\pi l}\right|^{2}+\left|\frac{\sin (\pi l(\tau-\Delta) / T)}{\pi l}\right|^{2} & \tau \in[\Delta, T+\Delta], l \neq 0\end{cases}
\end{aligned}
$$

where $\delta(l)$ is the Kronecker delta and $l=\left|m-m_{0}\right|$ is the spectral distance between the interfering subcarrier and the victim one.

According to (15), the OFDM interference power tables do not depend on the phase offset $\varphi$. In Table 3 , we give some examples of the instantaneous interference tables for $\tau=T / 4, T / 3$ and $T / 2$. These examples are also depicted in Figure 7 where we see that the interference level vary with respect to the timing offset $\tau$ and the spectral distance $l$.

\subsubsection{OFDM mean interference table}

In order to calculate the mean interference table $\bar{I}(l)$, we assume a timing offset $\tau$ uniformly distributed in $[0, T+$ $\Delta]$

$$
\bar{I}(l)=\int_{\tau} I(\tau, l) p(\tau) d \tau
$$

where $p(\tau)$ is the probability density function of the random variable $\tau$,

$$
p(\tau)=\left\{\begin{array}{lr}
1 /(T+\Delta) & \tau \in[0, T+\Delta] \\
0 & \text { elsewhere }
\end{array}\right.
$$

Substituting (15) and (17) into (16), we obtain

Table 3 OFDM instantaneous interference tables for $\tau=\{T / 4, T / 3, T / 2\}$

\begin{tabular}{cccc}
\hline $\boldsymbol{I}$ & $\boldsymbol{\tau}=\boldsymbol{T / 4}$ & $\boldsymbol{\tau}=\boldsymbol{T} / \mathbf{3}$ & $\boldsymbol{\tau}=\boldsymbol{T} \mathbf{2}$ \\
{$[\mathbf{d B}]$} & {$[\mathbf{d B}]$} & {$[\mathrm{dB}]$} \\
\hline 0 & -01.06 & -01.72 & -02.73 \\
1 & -15.29 & -11.27 & -07.63 \\
2 & -15.98 & -13.28 & -15.98 \\
3 & -17.18 & -17.17 & -24.83 \\
4 & -19.00 & -24.95 & -19.00 \\
5 & -21.62 & -38.96 & -29.27 \\
6 & -25.52 & -25.58 & -25.52 \\
\hline
\end{tabular}

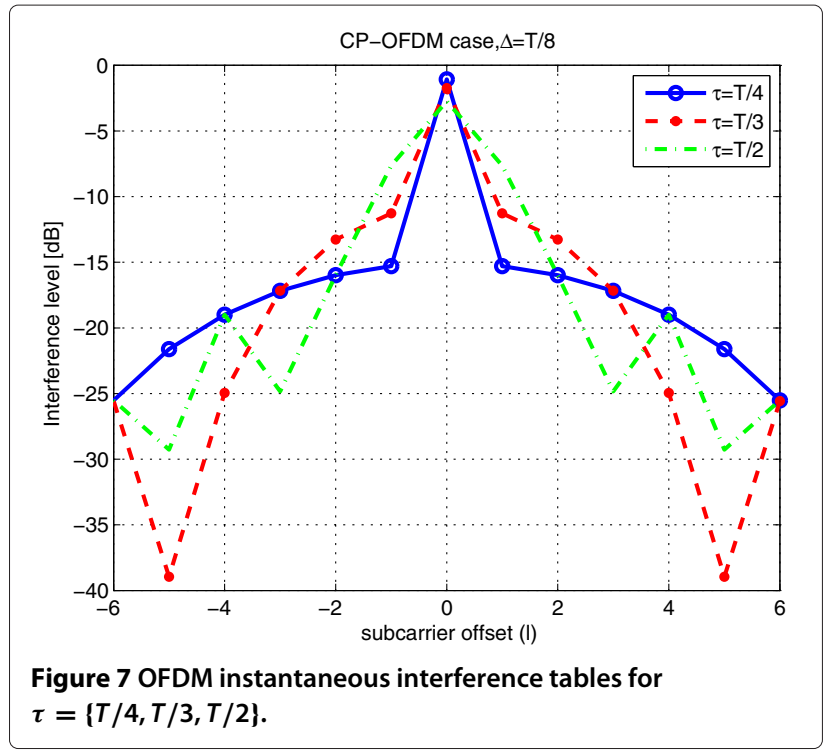

case $1(l=0)$

$$
\begin{aligned}
\bar{I}(0) & =\frac{1}{T+\Delta}\left[\int_{0}^{\Delta} d \tau+\int_{\Delta}^{T+\Delta} \frac{(T+\Delta-\tau)^{2}+(\tau-\Delta)^{2}}{T^{2}} d \tau\right] \\
& =\frac{2 T+3 \Delta}{3(T+\Delta)}
\end{aligned}
$$

case $2(l \neq 0)$

$$
\begin{aligned}
\bar{I}(l)= & \frac{1}{T+\Delta} \int_{\Delta}^{T+\Delta}\left|\frac{\sin (\pi l(T+\Delta-\tau) / T)}{\pi l}\right|^{2} \\
& +\left|\frac{\sin (\pi l(\tau-\Delta) / T)}{\pi l}\right|^{2} d \tau=\frac{T}{(\pi l)^{2}(T+\Delta)}
\end{aligned}
$$

The OFDM mean interference table is compared to the PSD-based OFDM interference table in Figure 8 and Table 4. We can see that the two interference levels computed by the PSD and the proposed interference tables are quite different. In fact, the bigger the spectral distance is, the greater the gap in interference level becomes. It must be noted that, except for $l=0$, the interference levels presented by the proposed model is higher than the PSD-based one.

\subsection{FBMC case}

In this scheme, the idea is to transmit offset quadrature amplitude modulation (OQAM) data symbols instead 


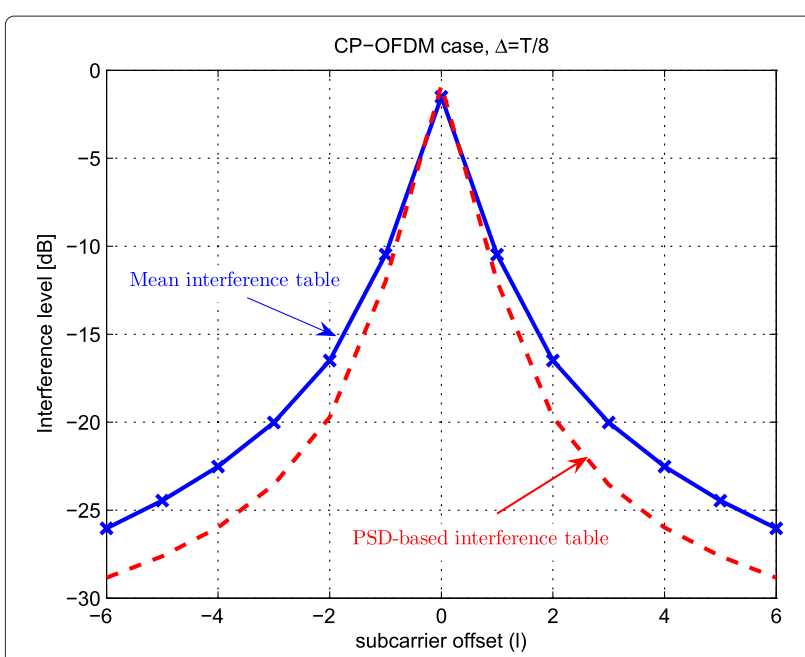

Figure 8 OFDM mean interference table vs. PSD-based OFDM interference table.

of conventional QAM ones, where the in-phase and the quadrature components are time staggered by half a symbol period, $T / 2$ [21]. The second specificity of this scheme is that considering two successive subcarriers, the time delay $T / 2$ is introduced into the imaginary part of the QAM symbols on one of the subcarriers, whereas it is introduced into the real part of the symbols on the other one [22,23]. It is worth noticing that the spacing between two successive subcarriers is $1 / T$.

According to [22], the continuous-time baseband FBMC transmit signal can be written

$$
s(t)=\sum_{m=0}^{N-1} \sum_{n=-\infty}^{+\infty} a_{m, n} \gamma_{m, n}(t),
$$

where $N$ is the number of subcarriers, $a_{m, n}$ are the realvalued transmitted data symbols and $\gamma_{m, n}$ is defined by

Table 4 OFDM mean interference table vs. PSD-based OFDM interference table

\begin{tabular}{lcc}
\hline $\boldsymbol{I}$ & $\begin{array}{c}\text { Mean interf. table } \\
{[\mathbf{d B}]}\end{array}$ & $\begin{array}{c}\text { PSD interf. table } \\
{[\mathbf{d B}]}\end{array}$ \\
\hline 0 & -01.51 & -00.87 \\
1 & -10.48 & -12.00 \\
2 & -16.50 & -19.72 \\
3 & -20.02 & -23.55 \\
4 & -22.52 & -25.98 \\
5 & -24.45 & -27.62 \\
6 & -26.04 & -28.83 \\
\hline
\end{tabular}

$$
\gamma_{m, n}(t)=g(t-n T / 2) e^{j \frac{2 \pi}{T} m t} e^{j \varphi_{m, n}}
$$

and with

$$
\varphi_{m, n}=\frac{\pi}{2}(n+m)-\pi n m
$$

According to (20), we can define the asynchronous signal coming from the interferer on the $m$-th subcarrier $s_{m}(t-\tau, \varphi)$ as follows:

$$
s_{m}(t-\tau, \varphi)=\sum_{n=-\infty}^{+\infty} a_{m, n} \gamma_{m, n}(t-\tau) e^{j \varphi} .
$$

The $m_{0}$-th output of the receiver filter on the $n_{0}$-th signalling interval (i.e., $\left.t=n_{0} T / 2\right)$ coming from $s_{m}(t-\tau, \varphi)$, will be

$$
\begin{aligned}
y_{m_{0}, n_{0}}(\tau, \varphi)= & \left\langle s_{m}(t-\tau, \varphi), \gamma_{m_{0}, n_{0}}(t)\right\rangle \\
= & \int_{-\infty}^{+\infty} s_{m}(t-\tau, \varphi) \gamma_{m_{0}, n_{0}}^{*}(t) d t .
\end{aligned}
$$

Substituting (23) and (21) in (24), the signal $y_{m_{0}, n_{0}}(\tau)$ becomes

$$
\begin{aligned}
y_{m_{0}, n_{0}}(\tau, \varphi)= & \sum_{n=-\infty}^{+\infty} a_{m, n} e^{j\left(\varphi+\varphi_{m, n}-\varphi_{m_{0}, n_{0}}\right)} e^{-j \frac{2 \pi}{T} m \tau} \int_{-\infty}^{+\infty} \\
& \times g(t-n T / 2-\tau) g\left(t-n_{0} T / 2\right) e^{j \frac{2 \pi}{T}\left(m-m_{0}\right) t} d t .
\end{aligned}
$$

In order to get simplified, easier-to-manipulate expressions, let us define the following integral

$$
\left.\Psi(t, \tau, l)\right|_{t=t_{1}} ^{t_{2}}=\int_{t_{1}}^{t_{2}} g(t-\tau) g(t) e^{j \frac{2 \pi}{T} l t} d t .
$$

If we consider the PHYDYAS prototype filter, the explicit form expressions of this integral are given in (27) and (28), respectively, for $l=0$ and $l \neq 0$. The details of calculus are given in Appendix A. 


$$
\begin{aligned}
& \left.\Psi(t, \tau, 0)\right|_{t=t_{1}} ^{t_{2}}=\int_{t_{1}}^{t_{2}} g(t-\tau) g(t) d t \\
& =\frac{t}{A}\left[1+2 \sum_{k=1}^{K-1} G_{k}^{2} \cos \left(\frac{2 \pi}{K T} k \tau\right)\right] \\
& +\frac{K T}{\pi A}\left\{\sum_{k=1}^{K-1} \sum_{k^{\prime}=1}^{K-1}(-1)^{k+k^{\prime}} \frac{G_{k} G_{k^{\prime}}}{k+k^{\prime}}\right. \\
& \times \sin \left(\frac{2 \pi}{K T}\left(\left(k+k^{\prime}\right) t-k \tau\right)\right) \\
& +\sum_{k=1}^{K-1} \sum_{\substack{k^{\prime}=1 \\
k \neq k^{\prime}}}^{K-1}(-1)^{k+k^{\prime}} \frac{G_{k} G_{k^{\prime}}}{k-k^{\prime}} \\
& \times \sin \left(\frac{2 \pi}{K T}\left(\left(k-k^{\prime}\right) t-k \tau\right)\right) \\
& +\sum_{k=1}^{K-1}(-1)^{k} \frac{G_{k}}{k}\left[\sin \left(\frac{2 \pi}{K T} k \tau\right)\right. \\
& \left.\left.+\sin \left(\frac{2 \pi}{K T} k(t-\tau)\right)\right]\right\}\left.\right|_{t=t_{1}} ^{t_{2}}, \\
& \left.\Psi(t, \tau, l)\right|_{t=t_{1}} ^{t_{2}}=\frac{T}{j 2 \pi l A} e^{j \frac{2 \pi}{T} l t}+\frac{K T}{j 2 \pi A} \sum_{k=1}^{K-1}(-1)^{k} G_{k} \\
& \times\left[\frac{1+e^{-j \frac{2 \pi}{K T} k \tau}}{k+K l} e^{j \frac{2 \pi}{K T}(k+K l) t}-\frac{1+e^{j \frac{2 \pi}{K T} k \tau}}{k-K l}\right. \\
& \left.\times e^{-j \frac{2 \pi}{K T}(k-K l) t}\right]+\frac{K T}{j 2 \pi A} \sum_{k=1}^{K-1} \sum_{k^{\prime}=1}^{K-1}(-1)^{k+k^{\prime}} G_{k} G_{k^{\prime}} \\
& \times\left[e^{-j \frac{2 \pi}{K T} k \tau}\left(\frac{e^{j \frac{2 \pi}{K T}\left(k+k^{\prime}+K l\right) t}}{k+k^{\prime}+K l}+\frac{e^{j \frac{2 \pi}{K T}\left(k-k^{\prime}+K l\right) t}}{k-k^{\prime}+K l}\right)\right. \\
& \left.-e^{j \frac{2 \pi}{K T} k \tau}\left(\frac{e^{-j \frac{2 \pi}{K T}\left(k+k^{\prime}-K l\right) t}}{k+k^{\prime}-K l}+\frac{e^{-j \frac{2 \pi}{K T}\left(k-k^{\prime}-K l\right) t}}{k-k^{\prime}-K l}\right)\right]\left.\right|_{t=t_{1}} ^{t_{2}},
\end{aligned}
$$

where $\lfloor\alpha\rfloor$ denotes the floor function (the largest integer less than or equal to $\alpha$ ).

3.2.2 Case $2:\left(\tau<\left(n_{0}-n\right) \frac{T}{2}\right)$

According also to (25) and (26), we obtain

$$
\begin{aligned}
y_{m_{0}, n_{0}}(\tau, \varphi)= & \sum_{n=-2 K+n_{0}+1}^{n_{0}+\left\lceil\frac{-\tau}{T / 2}\right\rceil-1} a_{m, n} e^{j\left(\varphi+\varphi_{m, n}-\varphi_{m_{0}, n_{0}}\right)} \\
& \times\left. e^{-j \frac{2 \pi}{T} m \tau} \Psi(t, \tau, l)\right|_{t=\left(n_{0}-n\right) \frac{T}{2}} ^{K T+\tau},
\end{aligned}
$$

where $\lceil\alpha\rceil$ is the ceil function (the smallest integer greater than or equal to $\alpha$ ).

After the OQAM decision, we can write the total complex symbol $y_{\text {tot }}(\tau, \varphi)$ as follows:

$$
y_{\text {tot }}(\tau, \varphi)=\Re\left\{y_{m_{0}, n_{0}}(\tau, \varphi)\right\}+j \Re\left\{y_{m_{0}, n_{0}+1}(\tau, \varphi)\right\},
$$

and the corresponding interference power table $I(\tau, l)$ can thus be given by the following expression:

$$
I(\tau, l)=\mathbb{E}_{a_{m, n}, \varphi}\left[\left|y_{t o t}(\tau, \varphi)\right|^{2}\right] .
$$

In Figures 9 and 10, PHYDYAS and IOTA interference tables $I(\tau, l)$ are plotted for different values of the timing offset $\tau=T / 4, T / 3, T / 2$. We also see that the interference varies with respect to the timing offset $\tau$ and the spectral distance $l$ between the interfering subcarrier and the victim one. Furthermore, PHYDYAS and IOTA mean interference tables are compared to the respective PSD-based interference tables in Figures 11 and 12, respectively. Looking at these figures, we find that the two models lead to different results. However, this difference is negligible when the interference level is high.

$$
\begin{aligned}
A & =\int_{0}^{K T}\left[1+2 \sum_{k=1}^{K-1}(-1)^{k} G_{k} \cos \left(\frac{2 \pi}{K T} k t\right)\right]^{2} d t \\
& =K T\left[1+2 \sum_{k=1}^{K-1} G_{k}^{2}\right] .
\end{aligned}
$$

\section{Asynchronous interference in frequency selective channels}

In this section, we refer to the system model shown in Figure 13. The asynchronous OFDM/FBMC transmit signal $s(t-\tau, \varphi)$ propagates through a frequency selective totype filter $g(t)$ can be non zero only when $t \in[0, K T]$ where $K$ represents its overlapping factor. Accordingly, the product $g(t) g(t-\tau)$ can be nonzero only when the 


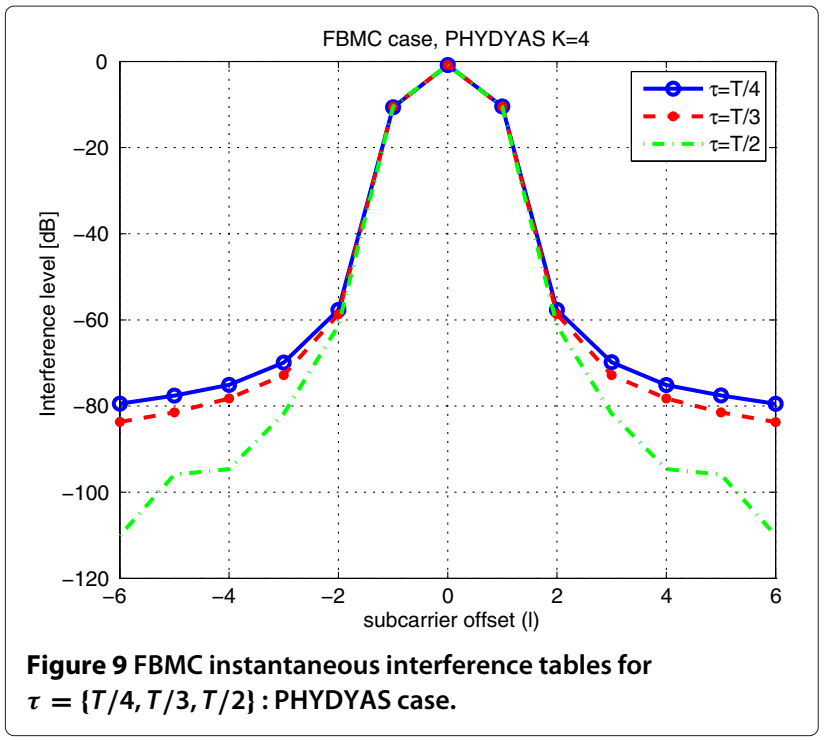

multipath channel, where its equivalent sample-spaced impulse response [24] is given by

$$
h(t)=\sum_{i=0}^{L-1} h_{i} \delta\left(t-\frac{n_{i}}{N} T\right),
$$

where $n_{0}<n_{1}<\ldots<n_{L-1}<C$ and $C$ is the maximum delay spread of the channel normalized by the sampling period $(T / N)$, and $h_{i}$ are the complex channel path gains, which are assumed mutually independent, where $\mathbb{E}\left[h_{i} h_{i}^{*}\right]=\gamma_{i}$, and $\mathbb{E}\left[h_{i} h_{j}^{*}\right]=0$ when $i \neq j$. We further assume that the power is normalized such that $\sum_{i=0}^{L-1} \gamma_{i}=1$.

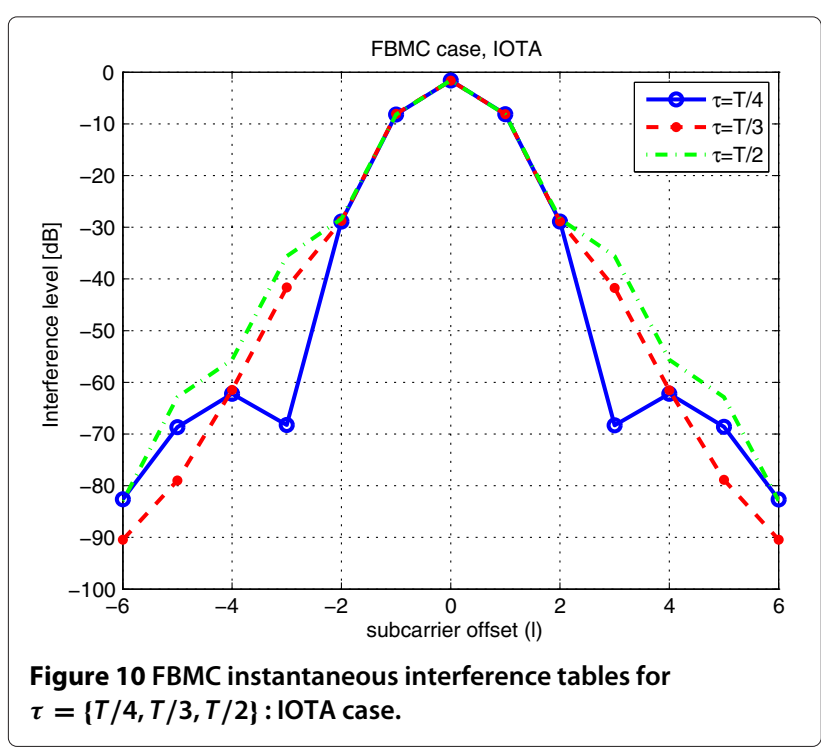

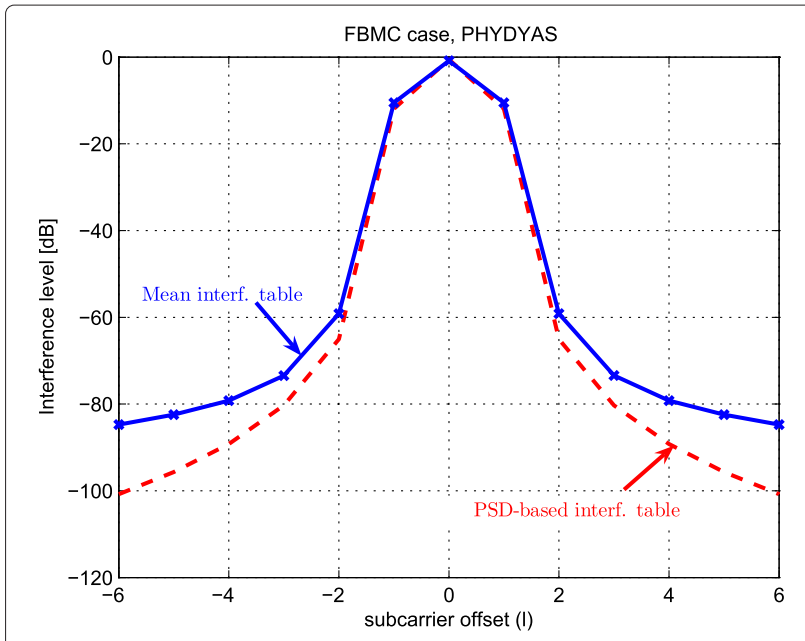

Figure 11 FBMC mean interference table vs. PSD-based FBMC interference table: PHYDYAS case.

In this case, the interference signal received at the input of the multicarrier demodulator $r(t-\tau, \varphi)$ is given by

$$
r(t-\tau, \varphi)=h(t) \star s(t-\tau, \varphi),
$$

where $\star$ stands for the convolution product.

In the following analysis, we investigate the effects of the propagation channel on the asynchronous interference signal coming from the $m$-th subcarrier $s_{m}(t-\tau, \varphi)$. In the following analysis, two cases will be investigated : CP-OFDM case and FBMC one.

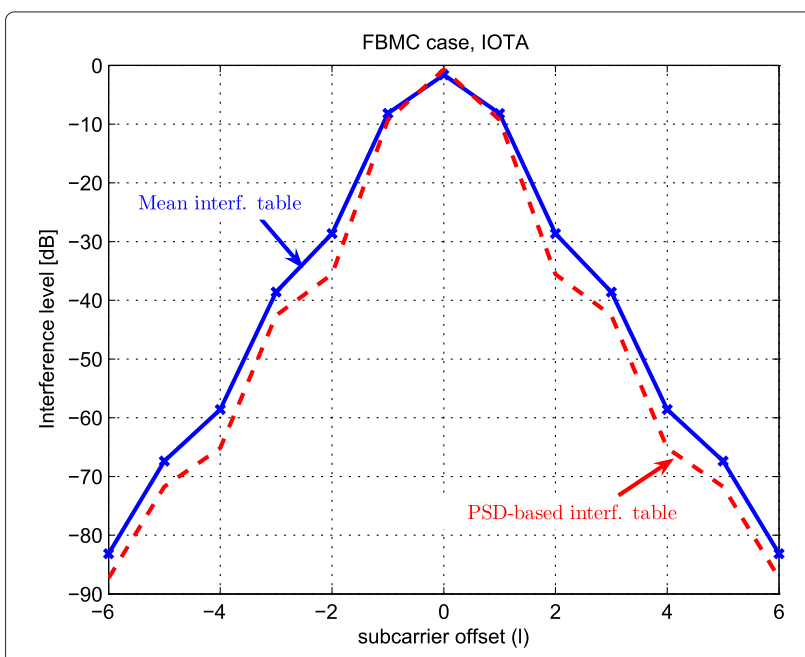

Figure 12 FBMC mean interference table vs. PSD-based FBMC interference table : IOTA case. 


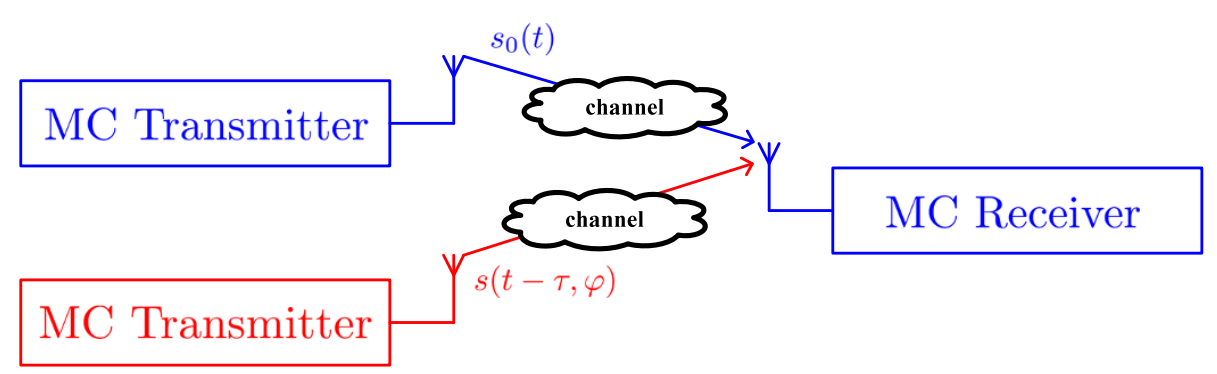

Figure 13 Asynchronous interference in the presence of multi-path effects.

\subsection{CP-OFDM case}

According to (8) and (35), the interference signal at the input of the OFDM receiver can be written as follows:

$$
\begin{aligned}
r(t-\tau, \varphi)= & h(t) \star s_{m}(t-\tau, \varphi)=\left(\sum_{i=0}^{L-1} h_{i} \delta\left(t-\frac{n_{i}}{N} T\right)\right) \\
& \star\left(\sum_{n=-\infty}^{n=+\infty} x_{m, n} f_{T}(t-n(T+\Delta)-\tau)\right. \\
& \left.\times e^{j\left[\frac{2 \pi}{T} m(t-n(T+\Delta)-\tau)+\varphi\right]}\right) \\
= & \sum_{i=0}^{L-1} \sum_{n=-\infty}^{+\infty} h_{i} x_{m, n} f_{T}\left(t-n(T+\Delta)-\tau-\frac{n_{i}}{N} T\right) \\
& \times e^{j\left[\frac{2 \pi}{T} m\left(t-n(T+\Delta)-\tau-\frac{n_{i}}{N} T\right)+\varphi\right] .}
\end{aligned}
$$

In weakly and mildly frequency selective channels, $\frac{n_{i}}{N} T$ is small enough. We can thus consider that $f_{T}(t-n(T+\Delta)-$ $\left.\tau-\frac{n_{i}}{N} T\right)=f_{T}(t-n(T+\Delta)-\tau)$. Hence, the signal $r(t-\tau, \varphi)$ becomes

$$
\begin{aligned}
r(t-\tau, \varphi)= & \sum_{i=0}^{L-1} h_{i} e^{j \frac{2 \pi n_{i}}{N} m} \sum_{n=-\infty}^{+\infty} x_{m, n} f_{T}(t-n(T+\Delta)-\tau) \\
& \times e^{j\left[\frac{2 \pi}{T} m(t-n(T+\Delta)-\tau)+\varphi\right]} \\
= & H(m) \sum_{n=-\infty}^{+\infty} x_{m, n} f_{T}(t-n(T+\Delta)-\tau) \\
& \times e^{j\left[\frac{2 \pi}{T} m(t-n(T+\Delta)-\tau)+\varphi\right]} \\
= & H(m) s_{m}(t-\tau, \varphi),
\end{aligned}
$$

where $H(m)=\sum_{i=0}^{L-1} h_{i} e^{j \frac{2 \pi n_{i}}{N} m}$ representing the complex channel gain at the $m$-th subcarrier.

Based on (37), (10) and (14), the $m_{0}$-th output of the receiver filter on the $n_{0}$-th signalling interval resulting from the received interference signal $r(t-\tau, \varphi)$ is expressed for both cases $0<\tau<\Delta$ and $\Delta<\tau<T+\Delta$ as follows:

\subsubsection{Case 1: $(0<\tau<\Delta)$}

$$
y_{m_{0}, n_{0}}(\tau, \varphi)= \begin{cases}H\left(m_{0}\right) x_{m_{0}, n_{0}} e^{-j\left[\frac{2 \pi}{T} m_{0} \tau-\varphi\right]} & m=m_{0} \\ 0 & \text { otherwise }\end{cases}
$$

\subsubsection{Case 2: $(\Delta<\tau<T+\Delta)$}

When $m \neq m_{0}$

$$
\begin{aligned}
y_{m_{0}, n_{0}}(\tau, \varphi)= & H(m) e^{-j\left[\frac{2 \pi}{T} m \tau-\varphi\right]}\left\{\frac{x_{m, n_{0}-1}}{\pi\left(m-m_{0}\right)} e^{-j \frac{2 \pi}{T} m(T+\Delta)}\right. \\
& \times e^{j \frac{\pi}{T}\left(m-m_{0}\right)(\tau+\Delta)} \sin \left[\pi\left(m-m_{0}\right)(\tau-\Delta) / T\right] \\
& +\frac{x_{m, n_{0}}}{\pi\left(m-m_{0}\right)} e^{j \frac{\pi}{T}\left(m-m_{0}\right)(T+\Delta+\tau)} \\
& \left.\times \sin \left[\pi\left(m-m_{0}\right)(T+\Delta-\tau) / T\right]\right\} .
\end{aligned}
$$

When $m=m_{0}$, the signal $y_{m_{0}, n_{0}}(\tau)$ is given by

$$
\begin{aligned}
y_{m_{0}, n_{0}}(\tau, \varphi)= & H\left(m_{0}\right) e^{-j\left[\frac{2 \pi}{T} m_{0} \tau-\varphi\right]}\left\{x_{m_{0}, n_{0}-1} e^{-j \frac{2 \pi}{T} m_{0}(T+\Delta)}\right. \\
& \left.\times \frac{\tau-\Delta}{T}+x_{m_{0}, n_{0}} \frac{T+\Delta-\tau}{T}\right\}
\end{aligned}
$$

Consequently, the resulting interference power $P_{\text {interf }}$ will be the product of the channel power gain of the interfering subchannel and the corresponding interference weight given in (3). Then, we write the interference power in $m_{0}$-th subchannel as

$$
P_{\text {interf }}\left(m_{0}, \tau\right)=P_{\text {trans }}(m) I\left(\tau,\left|m-m_{0}\right|\right)|H(m)|^{2},
$$

where,

- $P_{\text {trans }}(m)$ is the transmitted power on the subchannel $m$.

- $I\left(\tau,\left|m-m_{0}\right|\right)$ represents the interference weight for the timing offset $\tau$ and the spectral distance $\left|m-m_{0}\right|$.

- $|H(m)|^{2}$ is the channel power gain for subchannel $m$. 


\subsection{FBMC case}

Similarly, we express the interference signal received at the input of the FBMC receiver $r(t-\tau, \varphi)$. According to (23) and (35), we write

$$
\begin{aligned}
r(t-\tau, \varphi)= & h(t) \star s_{m}(t-\tau, \varphi)=\left(\sum_{i=0}^{L-1} h_{i} \delta\left(t-\frac{n_{i}}{N} T\right)\right) \\
& \star\left(\sum_{m=0}^{N-1} \sum_{n=-\infty}^{+\infty} a_{m, n} \gamma_{m, n}(t-\tau) e^{j \varphi}\right) \\
= & \sum_{i=0}^{L-1} \sum_{n=-\infty}^{+\infty} h_{i} a_{m, n} \gamma_{m, n}\left(t-\tau-\frac{n_{i}}{N} T\right) e^{j \varphi}
\end{aligned}
$$

Substituting (21) in (42), we obtain

$$
\begin{aligned}
r(t-\tau, \varphi)= & \sum_{i=0}^{L-1} \sum_{n=-\infty}^{+\infty} h_{i} a_{m, n} g\left(t-n T / 2-\tau-\frac{n_{i}}{N} T\right) \\
& \times e^{j \frac{2 \pi}{T} m\left(t-\tau-\frac{n_{i}}{N} T\right)} e^{j \varphi}=\sum_{i=0}^{L-1} h_{i} e^{-j \frac{2 \pi}{N} m n_{i}} \\
& \sum_{n=-\infty}^{+\infty} a_{m, n} g\left(t-n T / 2-\tau-\frac{n_{i}}{N} T\right) e^{j \frac{2 \pi}{T} m(t-\tau)} e^{j \varphi} .
\end{aligned}
$$

We can notice that $g\left(t-n T / 2-\tau-\frac{n_{i}}{N} T\right)$ may have relatively slow variations when $\frac{n_{i}}{N} T \in\left[0, \tau_{\mathrm{ds}}\right]\left(\tau_{\mathrm{ds}}\right.$ is the maximum delay spread of the channel) $[25,26]$. Indeed, compared to the coherence bandwidth $B_{c}$, the filter bandwidth is very small, which also means that the time variations of the prototype filter $g(t)$ are necessarily limited.

Consequently, the signal $r(t-\tau, \varphi)$ becomes

$$
\begin{aligned}
r(t-\tau, \varphi)= & \sum_{i=0}^{L-1} h_{i} e^{-j \frac{2 \pi}{N} m n_{i}} \sum_{n=-\infty}^{+\infty} a_{m, n} g(t-n T / 2-\tau) \\
& \times e^{j \frac{2 \pi}{T} m(t-\tau)} e^{j \varphi}=H(m) s_{m}(t-\tau, \varphi) .
\end{aligned}
$$

Now, let $\varphi_{H(m)}$ be the phase angle of the complex channel gain $H(m)$, i.e.,

$$
H(m)=|H(m)| e^{j \varphi_{H}(m)} .
$$

Hence, we can write $r(t-\tau, \varphi)$ as follows:

$$
r(t-\tau, \varphi)=|H(m)| s_{m}\left(t-\tau, \varphi+\varphi_{H(m)}\right) .
$$

According (32) and (45), the output signal after the OQAM decision is given by

$$
y_{\text {tot }}^{\prime}(\tau, \varphi)=|H(m)| y_{\text {tot }}\left(\tau, \varphi+\varphi_{H(m)}\right),
$$

where, $y_{\text {tot }}(\tau, \varphi)$ is given in (32).
Now, let $\varphi$ and $\varphi^{\prime}$ be two uniform random variables defined in the following intervals $\varphi \in[0,2 \pi]$ and $\varphi^{\prime} \in$ $[\alpha, \alpha+2 \pi]$, respectively. We notice that

$$
\mathbb{E}_{a_{m, n}, \varphi}\left[\left|y_{t o t}(\tau, \varphi)\right|^{2}\right]=\mathbb{E}_{a_{m, n}, \varphi^{\prime}}\left[\left|y_{t o t}\left(\tau, \varphi^{\prime}\right)\right|^{2}\right] .
$$

Therefore, the corresponding interference power $P_{\text {interf }}$ is also the product of the channel power gain of the interfering subcarrier $|H(m)|^{2}$ and the corresponding interference weight given in (33). Thus, the interference power at the $m_{0}$-th subchannel is given by

$$
P_{\text {interf }}\left(m_{0}, \tau\right)=P_{\text {trans }}(m) I\left(\tau,\left|m-m_{0}\right|\right)|H(m)|^{2} .
$$

In general, the asynchronous interference power arriving through a frequency selective channel can be calculated using the following expression:

$$
P_{\text {interf }}\left(m_{0}, \tau\right)=d^{-\beta} P_{\text {trans }}(m) I\left(\tau,\left|m-m_{0}\right|\right)|H(m)|^{2},
$$

where,

- $d$ is the distance between the interferer and the victim user.

- $\beta$ is the path loss exponent.

- $P_{\text {trans }}(m)$ is the transmitted power on the interfering subchannel $m$.

- $I\left(\tau,\left|m-m_{0}\right|\right)$ is the interference weight for the timing offset $\tau$ and the spectral distance $\left|m-m_{0}\right|$.

- $|H(m)|^{2}$ is the channel power gain between the interfering transmitter and the victim receiver on subchannel $m$.

In the next section, we investigate the accuracy of the proposed interference modeling expressed by Equation 48 . Various applications and scenarios can be studied using this interference model.

\section{Simulation results}

In this section, we consider the uplink transmission in OFDM/FBMC-based network depicted in Figure 14a. The reference mobile user $\mathrm{MU}_{0}$ and the interfering one $\mathrm{MU}_{1}$ communicating respectively with $\mathrm{BS}_{0}$ and $\mathrm{BS}_{1}$. Moreover, $M U_{0}$ and $M U_{1}$ are respectively located at distances $d_{0}$ and $d$ from the reference base station $\mathrm{BS}_{0}$. It is assumed that, the transmitted power of each user must guarantee a target signal to noise ration $\mathrm{SNR}_{\mathrm{t}}=20 \mathrm{~dB}$ at its base station $\left(\mathrm{BS}_{0}\right.$ for $\mathrm{MU}_{0}$ and $\mathrm{BS}_{1}$ for $M \mathrm{MU}_{1}$ ).

Concerning the frequency scheme, the subcarriers are allocated according to the scheme described in Figure 14b where, the size of each subcarrier block is set at 18 subcarriers. Here, we have chosen the practical size of subcarrier block in WiMax 802.16 [27].

All signals propagate through different multipath channels using a similar propagation model, where the impulse responses of the multipath channel between $\mathrm{MU}_{0}, \mathrm{MU}_{1}$ 

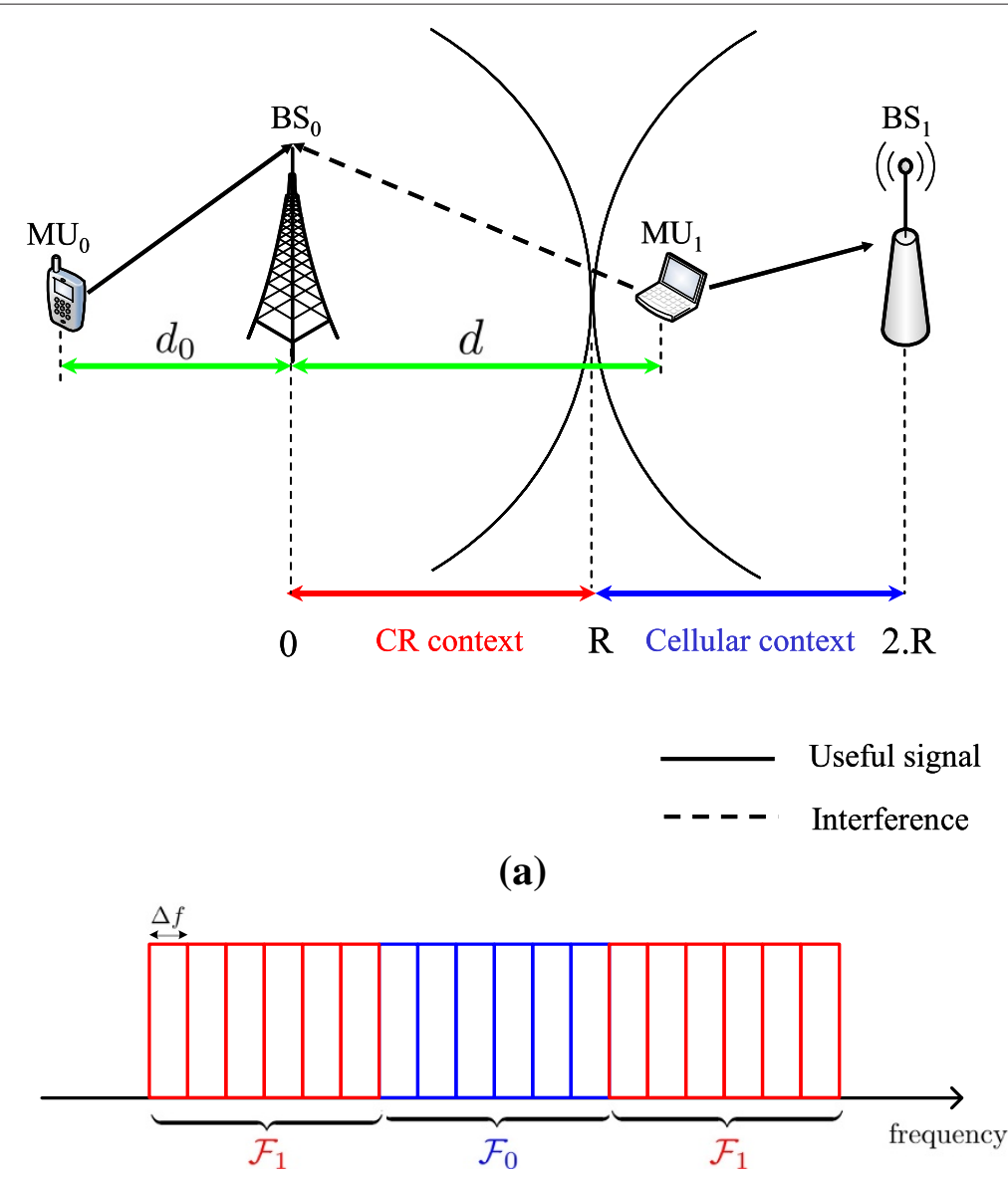

(b)

Figure 14 Interference model. (a) The reference user coexists with an asynchronous interferer. (b) Subcarrier assignment for both coexisting systems.

and the reference base station $\mathrm{BS}_{0}$ are denoted by $h_{0}$ and $h_{1}$, respectively. The considered model is the Pedestrian-A model whose parameters are given in Table 5 . The choice of this model is based on the assumption that the subcarriers of interest experience flat fading. Therefore, the interference caused by the multipath effects are negligible compared to the one caused by time asynchronism, in the FBMC case.

Furthermore, the underlying channel model includes path loss effects which takes into account the position of the mobile user with respect to the reference base station $\mathrm{BS}_{0}$. The path loss of a received signal at distance $d$ is governed by the following expression [28] corresponding to a

Table 5 Channel parameters used in simulations

\begin{tabular}{lc}
\hline Parameter & Value \\
\hline Pedestrian-A relative delay & $0,110,190,410] \mathrm{ns}$ \\
Pedestrian-A average power & {$[0,-9.7,-19.2,-22.8] \mathrm{dB}$}
\end{tabular}

path loss exponent $\beta=3.76$ and a carrier frequency of 2 GHZ

$$
\Gamma_{\operatorname{loss}}(d)=128.1+37.6 \log _{10}(d[k m])[d B] .
$$

On the other hand, we consider a system with $N=1,024$ subcarriers and a sampling frequency of $10 \mathrm{MHz}$. The noise term is considered as a thermal noise with spectral density $N_{0}=-174 \mathrm{dBm} / \mathrm{Hz}$.

Each mobile user is assumed to be perfectly synchronized with its corresponding base station but it is not synchronized with the other base station. Because of the timing misalignment between $\mathrm{MU}_{1}$ and $\mathrm{BS}_{0}$, the signal arriving from $\mathrm{MU}_{1}$ at $\mathrm{BS}_{0}$ appears non-orthogonal to the desired signal arriving from $\mathrm{MU}_{0}$. This nonorthogonality generates interference and degrades the signal-to-interference-plus-noise ratio (SINR). Using the interference tables introduced previously, the instanta- 
neous SINR on a given subcarrier $m \in \mathcal{F}_{0}$ can be expressed as

$$
\begin{aligned}
& \operatorname{SINR}(m) \\
& =\frac{d_{0}^{-\beta} P_{\text {trans }}(m)\left|H_{0}(m)\right|^{2}}{\sum_{m^{\prime} \in \mathcal{F}_{1}} d^{-\beta} P_{\text {trans }}\left(m^{\prime}\right) I\left(\tau,\left|m^{\prime}-m\right|\right)\left|H_{1}\left(m^{\prime}\right)\right|^{2}+N_{0} \Delta f},
\end{aligned}
$$

where $\Delta f$ is the subcarrier spacing. Actually, the SINR expression given in (49) is established assuming the absence of ISI and ICI terms. Such an assumption is valid in the Pedestrian-A channel model.

Here, it is worth mentioning that the interference weight $I$ is computed by two methods: PSD-based interference tables and our proposed interference tables.

The objective of this section is to evaluate the average SINR and the spectral efficiency expressed, respectively by

$$
\begin{aligned}
& \operatorname{SINR}_{\text {average }}(m)=\mathbb{E}[\operatorname{SINR}(m)], \\
& C_{\text {average }}(m)=\mathbb{E}\left[\log _{2}(1+\operatorname{SINR}(m))\right],
\end{aligned}
$$

where $\mathbb{E}[$.$] stands for the statistical expectation which$ is computed over all channel realizations $\left(H_{0}(m)\right.$, $\left.\left\{H_{1}\left(m^{\prime}\right), m^{\prime} \in \mathcal{F}_{1}\right\}\right)$ and all values of the timing offset $\tau$ which is uniformly distributed over $[0, T]$.

Two different contexts will be analyzed as depicted in Figure 14:

- The classical multi-cellular context: when $d$ varies from $R$ to $2 R$, i.e., $M_{1}$ can move from the edge to the center of cell 1

- The cognitive radio context: when $d$ varies from 0 to $2 R$, i.e., $\mathrm{MU}_{1}$ can be very close to $\mathrm{BS}_{0}$ while transmitting to $\mathrm{BS}_{1}$.

It is worth mentioning that, in cognitive radio scenarios, we cannot always assume that both primary and secondary users are using OFDM or FBMC. However, the objective here is to highlight the potential gain that can be achieved when both primary and cognitive systems are using either OFDM or FBMC waveform.

In Figures 15, 16, and 17, we investigate, respectively, the accuracy of the SINR expression in the CP-OFDM, FBMC-PHYDYAS, and FBMC-IOTA cases. The averaged SINRs over all subcarriers $m \in \mathcal{F}_{0}$ are plotted against the distance $d$, using the instantaneous tables (line), the PSDbased table (point markers), and numerical simulation (cross markers). The instantaneous table results depicted in these figures show a perfect match to the corresponding simulation results. However, the PSD-based method

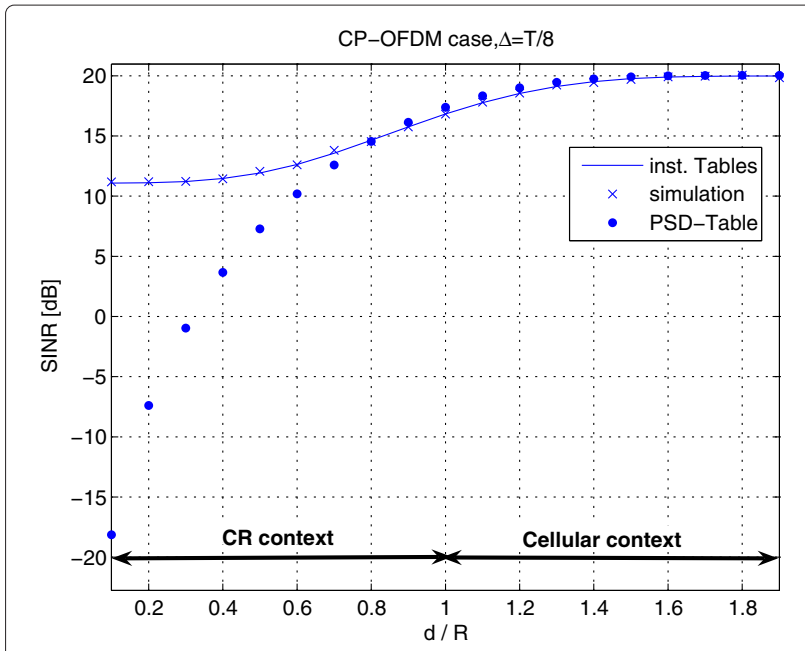

Figure 15 CP-OFDM average SINR vs. distance between $\mathrm{MU}_{1}$ and $\mathrm{BS}_{0}, \tau \in[0, T]$.

exhibits a strong inaccuracy especially in the cognitive radio context.

In the cognitive radio context, we observe a significant degradation of the OFDM SINR with respect to the target SNR $(20 \mathrm{~dB})$. Such a result can be explained by the high level of OFDM asynchronous interference caused by the timing misalignment which damages the orthogonality between the subcarriers. On the other hand, we notice a slight loss of the FBMC SINR with respect also to the target SNR of $20 \mathrm{~dB}$. The better performance of PHYDYAS-FBMC compared to IOTA-FBMC and CP-OFDM can be justified by the fact that only the two subcarriers on the edge of the cluster (subcarrier block) $\mathcal{F}_{0}$ suffers from the interference caused by their immediate adjacent subcarriers in $\mathcal{F}_{1}$ as depicted

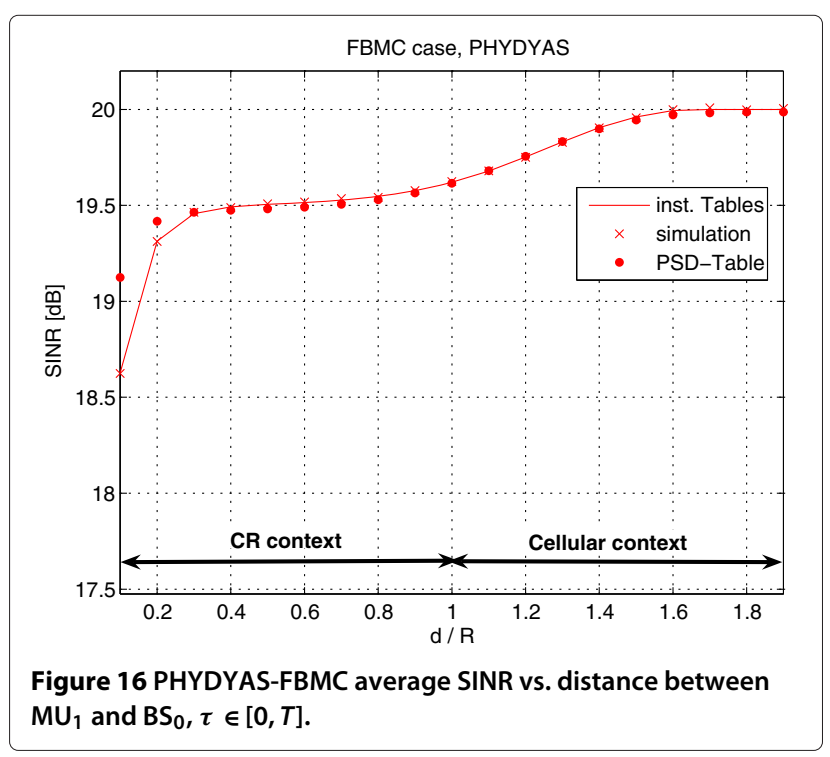




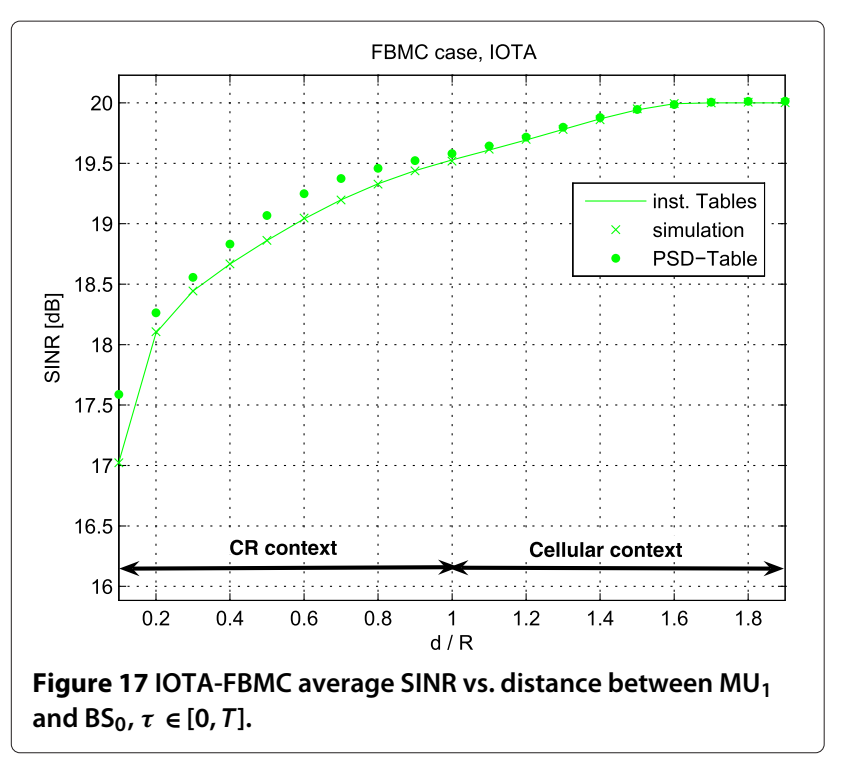

in Figure 11; whereas in IOTA-FBMC, two subcarriers at each edge are affected by the asynchronous interference coming from the two neighboring subcarriers at each edge as shown in Figure 12. Furthermore, the entire cluster $\mathcal{F}_{0}$ suffers, in the CP-OFDM case, from the asynchronous interference caused by all subcarriers of $\mathcal{F}_{1}$ (see Figure 8).

In the cellular context, the asynchronous interferer $\mathrm{MU}_{1}$ is quite far from the reference base station $\mathrm{BS}_{0}$ and, at the same time, it is close to its base station $\mathrm{BS}_{1}$. This means that its transmitted power is reduced and consequently the interference power received by $\mathrm{BS}_{0}$ will be much lower due to the path loss effect ( $d$ is quite large). Therefore, the impact of the asynchronous interference is less significant in the cellular context for all waveforms. Also, it is worth mentioning that SINRs of OFDM and FBMC converge to the target $\mathrm{SNR}(20 \mathrm{~dB})$ when $\mathrm{MU}_{1}$ is very far from $\mathrm{BS}_{0}$ as the interference becomes negligible compared to the noise level.

The impact of the asynchronous interference on the average spectral efficiency has also been investigated. Figure 18 shows the average spectral efficiency over all subcarriers $m \in \mathcal{F}_{0}$ against the distance $d$, for CPOFDM (solid line -), FBMC-PHYDYAS (dashed-dotted line -.), and FBMC-IOTA (dashed line --). Also, the accuracy of instantaneous and PSD-based tables is examined. Figure 18 shows that timing synchronization errors cause a degradation of the spectral efficiency. The same remarks can be formulated, here, where the FBMC system still outperforms the OFDM system. Furthermore, simulation and instantaneous tables results shown in Figure 18 validate the accuracy of the proposed interference model while the PSD-based tables still present a strong inaccuracy with respect to simulation results especially in the

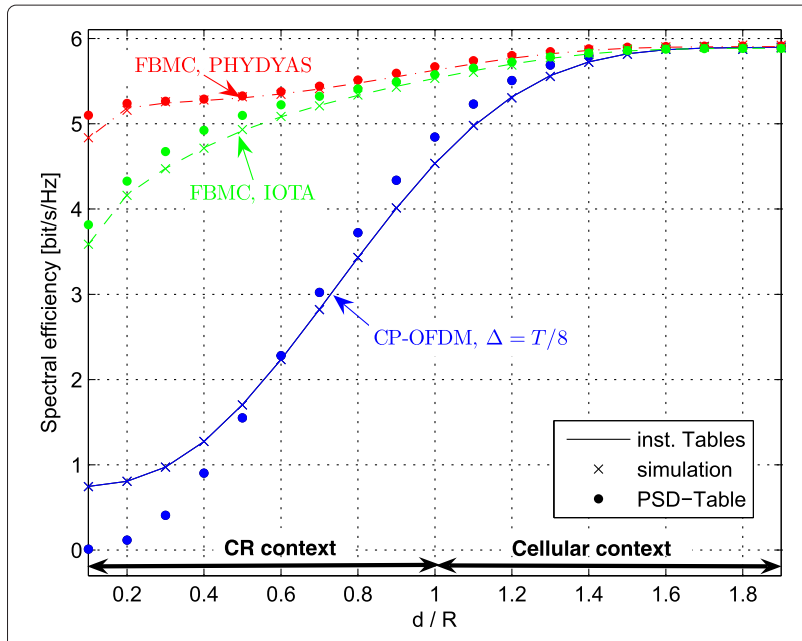

Figure 18 Spectral efficiency vs. distance between $\mathrm{MU}_{1}$ and $\mathrm{BS}_{0}$, $\tau \in[0, T]$.

cognitive radio context where the noise level becomes negligible compared to the asynchronous interference caused by $\mathrm{MU}_{1}$. We see that both modulation schemes (OFDM and FBMC) lead to identical spectral efficiency floor when $\mathrm{MU}_{1}$ is close to its base station because of the predominance of the noise term. We have to remind that the actual bit rate is lower for CP-OFDM because of the redundancy introduced by the cyclic prefix in each OFDM block.

\section{Conclusion}

In this paper, we have investigated the asynchronous interference modeling in OFDM and FBMC systems. First, the general concept of interference tables has been introduced where we have derived the PSD-based interference tables of CP-OFDM and FBMC for two considered waveforms: PHYDYAS and IOTA. It has been noticed that the PSD-based tables do not consider the timing offset between the interferer and the victim user.

Next, we have proposed new interference tables that model the correlation between a given interfering subcarrier and the victim one, not only as a function of the spectral distance separating both subcarriers but also with respect to the timing misalignment between the subcarrier holders. Theoretical expressions of these tables have been derived for both OFDM and FBMC systems.

The interference analysis has been extended to the case of frequency selective environments where we have proposed a table-based estimation method as a computationally simpler alternative to the numerical evaluation; as the latter requires huge computational efforts.

Furthermore, the accuracy of the proposed model has been validated through different simulation results, where 
the results based on the instantaneous tables method shows an excellent match with the corresponding simulation ones. In contrast to the instantaneous interference tables, we have shown through this evaluation that the PSD modeling exhibits a strong inaccuracy with respect to the numerical results.

Finally, through this evaluation, we have shown that in OFDM case, timing asynchronism between coexisting systems cause a severe degradation in the performance. This result is explained by the loss of orthogonality between all system subcarriers. In contrast to the OFDM system, the FBMC waveforms are demonstrated to be less sensitive to the timing misalignment between the cohabiting systems due to the better frequency localization of the prototype filter. The obtained results make FBMC a promising candidate for the physical layer of future cognitive radio systems.

\section{Appendix}

Proof of the expressions (27) and (28)

Let us first recall the impulse response of the PHYDYAS prototype filter which is given in [29],

$$
g(t)= \begin{cases}\frac{1}{\sqrt{A}}\left[1+2 \sum_{k=1}^{K-1}(-1)^{k} G_{k} \cos \left(\frac{2 \pi}{K T} k t\right)\right] & t \in[0, K T] \\ 0 & \text { elsewhere }\end{cases}
$$

where $A$ is the normalization factor

$$
\begin{aligned}
A & =\int_{0}^{K T}\left[1+2 \sum_{k=1}^{K-1}(-1)^{k} G_{k} \cos \left(\frac{2 \pi}{K T} k t\right)\right]^{2} d t \\
& =K T\left[1+2 \sum_{k=1}^{K-1} F_{k}^{2}\right] .
\end{aligned}
$$

Substituting the expression (52) in (26), we obtain when $l=0$

$$
\begin{aligned}
\left.\Psi(t, \tau, 0)\right|_{t=t_{1}} ^{t_{2}} & =\int_{t_{1}}^{t_{2}} g(t-\tau) g(t) d t \\
= & \int_{t_{1}}^{t_{2}}\left[1+2 \sum_{n=1}^{K-1}(-1)^{n} G_{n} \cos \left(\frac{2 \pi}{K T} n t\right)\right] \\
& \times\left[1+2 \sum_{n=1}^{K-1}(-1)^{n} G_{n} \cos \left(\frac{2 \pi}{K T} n(t-\tau)\right)\right] d t .
\end{aligned}
$$

Using some trigonometric transformations, the integral $\left.\Psi(t, \tau, 0)\right|_{t=t_{1}} ^{t_{2}}$ can be written in the following form:

$$
\begin{aligned}
\left.\Psi(t, \tau, 0)\right|_{t=t_{1}} ^{t_{2}}= & \int_{t_{1}}^{t_{2}} 1+2 \sum_{n=1}^{K-1}(-1)^{n} G_{n}\left[\cos \left(\frac{2 \pi}{K T} n t\right)\right. \\
& \left.+\cos \left(\frac{2 \pi}{K T} n(t-\tau)\right)\right]+2 \sum_{n=1}^{K-1} \sum_{m=1}^{K-1}(-1)^{n+m} \\
& \times G_{n} G_{m}\left[\cos \left(\frac{2 \pi}{K T}((n+m) t-n \tau)\right)\right. \\
& \left.+\cos \left(\frac{2 \pi}{K T}((n-m) t-n \tau)\right)\right] d t
\end{aligned}
$$

After integration, we get

$$
\begin{aligned}
\left.\Psi(t, \tau, 0)\right|_{t=t_{1}} ^{t_{2}} & \frac{t}{A}\left[1+2 \sum_{n=1}^{K-1} G_{n}^{2} \cos \left(\frac{2 \pi}{K T} n \tau\right)\right]+\frac{K T}{\pi A} \\
& \times\left\{\sum_{n=1}^{K-1} \sum_{m=1}^{K-1}(-1)^{n+m} \frac{G_{n} G_{m}}{n+m}\right. \\
& \times \sin \left(\frac{2 \pi}{K T}((n+m) t-n \tau)\right)+\sum_{n=1}^{K-1} \\
& \sum_{\substack{m=1 \\
n \neq m}}^{K-1}(-1)^{n+m} \frac{G_{n} G_{m}}{n-m} \sin \left(\frac{2 \pi}{K T}((n-m) t-n \tau)\right) \\
& +\sum_{n=1}^{K-1}(-1)^{n} \frac{G_{n}}{n}\left[\sin \left(\frac{2 \pi}{K T} n \tau\right)\right. \\
& \left.\left.+\sin \left(\frac{2 \pi}{K T} n(t-\tau)\right)\right]\right\}\left.\right|_{t=t_{1}} ^{t_{2}} .
\end{aligned}
$$

When $l \neq 0$,

$$
\begin{aligned}
\left.\Psi(t, \tau, l)\right|_{t=t_{1}} ^{t_{2}}= & \int_{t_{1}}^{t_{2}} g(t-\tau) g(t) e^{j \frac{2 \pi}{T} l t} d t \\
= & \int_{t_{1}}^{t_{2}} e^{j \frac{2 \pi}{T} l t}\left\{1+2 \sum_{n=1}^{K-1}(-1)^{n} G_{n}\right. \\
& \times\left[\cos \left(\frac{2 \pi}{K T} n t\right)+\cos \left(\frac{2 \pi}{K T} n(t-\tau)\right)\right] \\
& +2 \sum_{n=1}^{K-1} \sum_{m=1}^{K-1}(-1)^{n+m} G_{n} G_{m} \\
& \times\left[\cos \left(\frac{2 \pi}{K T}((n+m) t-n \tau)\right)\right. \\
& \left.\left.+\cos \left(\frac{2 \pi}{K T}((n-m) t-n \tau)\right)\right]\right\} d t .
\end{aligned}
$$


After integration, we obtain

$$
\begin{aligned}
\left.\Psi(t, \tau, l)\right|_{t=t_{1}} ^{t_{2}}= & \frac{T}{j 2 \pi l A} e^{j \frac{2 \pi}{T} l t} \\
& +\frac{K T}{j 2 \pi A} \sum_{n=1}^{K-1}(-1)^{n} G_{n}\left[\frac{1+e^{-j \frac{2 \pi}{K T} n \tau}}{n+K l}\right. \\
& \left.\times e^{j \frac{2 \pi}{K T}(n+K l) t}-\frac{1+e^{j \frac{2 \pi}{K T} n \tau}}{n-K l} e^{-j \frac{2 \pi}{K T}(n-K l) t}\right] \\
& +\frac{K T}{j 2 \pi A} \sum_{n=1}^{K-1} \sum_{m=1}^{K-1}(-1)^{n+m} G_{n} G_{m}\left[e^{-j \frac{2 \pi}{K T} n \tau}\right. \\
& \times\left(\frac{e^{j \frac{2 \pi}{K T}(n+m+K l) t}}{n+m+K l}+\frac{e^{j \frac{2 \pi}{K T}(n-m+K l) t}}{n-m+K l}\right)-e^{j \frac{2 \pi}{K T} n \tau} \\
& \left.\times\left(\frac{e^{-j \frac{2 \pi}{K T}(n+m-K l) t}}{n+m-K l}+\frac{e^{-j \frac{2 \pi}{K T}(n-m-K l) t}}{n-m-K l}\right)\right]\left.\right|_{t=t_{1}} ^{t_{2}} .
\end{aligned}
$$

\section{Competing interests}

The authors declare that they have no competing interests.

\section{Author details}

${ }^{1}$ ICTEAM, Université catholique de Louvain, Place du Levant, 2, Louvain-la-Neuve 1348, Belgium. ${ }^{2}$ CNAM - CEDRIC/LAETITIA, 292 rue Saint-Martin, Paris 75003, France.

\section{Acknowledgments}

This work has been partially supported by European Commission through the Emphatic project (ICT-318362)

\section{Received: 1 December 2013 Accepted: 7 April 2014}

\section{Published: 23 April 2014}

\section{References}

1. J Mitola J, Cognitive radio: an integrated agent architecture for software defined radio. PhD thesis, Royal Institute of Technology, Stockholm, Sweden, 2000

2. M Bellanger, Physical layer for future broadband radio systems, in IEEE Radio and Wireless Symposium (RWS), 2010 (IEEE Piscataway, 2010), pp. 436-439

3. C Stevenson, G Chouinard, Z Lei, W Hu, SJ Shellhammer, WM Caldwell, IEEE 802.22: the first cognitive radio wireless regional area network standard. IEEE Comm. Mag. 47(1), 130-138 (2009)

4. B Le Floch, M Alard, C Berrou, Coded orthogonal frequency division multiplex [TV broadcasting]. Proc. IEEE. 83(6), 982-996 (1995)

5. B Farhang-Boroujeny, OFDM versus filter bank multicarrier. IEEE Signal Process. Mag. 28(3), 92-112 (2011)

6. M Alard, Construction of a multicarrier signal (Patent WO/1996/035278) (1996). Available in WIPO: http://patentscope.wipo.int/search/en/ WO1996035278. Accessed Nov 2013

7. R Zakaria, D Le Ruyet, A novel filter-bank multicarrier scheme to mitigate the intrinsic interference: application to MIMO systems. IEEE Trans. Wireless Commun. 11(3), 1112-1123 (2012)

8. LG Baltar, DS Waldhauser, JA Nossek, Out-of-band radiation in multicarrier systems: a comparison, in MultiCarrier Spread Spectrum, vol. 1 (Springer Netherlands, 2007), pp. 107-116

9. H Zhang, DL Ruyet, M Terré, Spectral efficiency comparison between OFDM/OQAM and OFDM based CR networks. Wireless Commun. Mobile Comput. Wiley. 9, 1487-1501 (2009)

10. P Siohan, C Roche, Cosine-modulated filterbanks based on extended Gaussian functions. IEEE Trans. Signal Process. 48(11), 3052-3061

11. M Bellanger, D Le Ruyet, D Roviras, M Terré, J Nossek, L Baltar, Q Bai, D Waldhauser, M Renfors, T Ihalainen, A Viholainen, TH Stitz, J Louveaux, A
Ikhlef, V Ringset, H Rustad, M Najar, C Bader, M Payaro, D Katselis, E Kofidis, L Merakos, A Merentitis, N Passas, A Rontogiannis, S Theodoridis, D Triantafyllopoulou, D Tsolkas, D Xenakis, M Tanda, et al., FP7-ICT PHYDYAS - Physical layer for dynamic spectrum access and cognitive radio. http:// www.ict-phydyas.org. Accessed Nov 2013

12. M Bellanger, Specification and design of a prototype filter for filter bank based multicarrier transmission, in Proceedings of IEEE Int. Conf. on Acoustics, Speech, and Signal Processing, 2001. (ICASSP '01), vol. 4 (2001) (IEEE Piscataway, NJ, 2001), pp. 2417-2420

13. TWeiss, J Hillenbrand, A Krohn, FK Jondral, Mutual interference in OFDM-based spectrum pooling systems, in IEEE 59th Vehicular Technology Conference, vol. 4 (IEEE Piscataway, 2004), pp. 1873-1877

14. M Shaat, $F$ Bader, An uplink resource allocation algorithm for OFDM and FBMC based cognitive radio systems, in Proceedings of the Fifth International Conference On Cognitive Radio Oriented Wireless Networks Communications (CROWNCOM), 2010 (IEEE Piscataway, 2010), pp. 1-6

15. T Fusco, A Petrella, M Tanda, Sensitivity of multi-user filter-bank multicarrier systems to synchronization errors, in Communications, Control and Signal Processing, 2008. ISCCSP 2008. 3rd International Symposium On (IEEE Piscataway, 2008), pp. 393-398

16. H Saeedi-Sourck, Y Wu, JWM Bergmans, S Sadri, B Farhang-Boroujeny, Complexity and performance comparison of filter bank multicarrier and ofdm in uplink of multicarrier multiple access networks. IEEE Trans. Signal Process. 59(4), 1907-1912 (2011)

17. M Khodjet-Kesba, C Saber, D Roviras, Y Medjahdi, Multicarrier interference evaluation with jointly non-linear amplification and timing errors, in Vehicular Technology Conference (VTC Spring), 2011 IEEE 73rd (IEEE Piscataway, NJ, 2011), pp. 1-5

18. Y Medjahdi, D Le Ruyet, D Roviras, H Shaiek, R Zakaria, On the impact of the prototype filter on fbmc sensitivity to time asynchronism, in Wireless Communication Systems (ISWCS), 2012 International Symposium On (IEEE Piscataway, 2012), pp. 939-943

19. D5.1 D, Prototype Filter and Structure Optimization. Technical report, European project ICT-211887 PHYDYAS (2009)

20. H Zhang, DL Ruyet, D Roviras, Y Medjahdi, H Sun, Spectral efficiency comparison of OFDM/FBMC for uplink cognitive radio networks. EURASIP J. Adv. Signal Process (2010)

21. B Saltzberg, Performance of an efficient parallel data transmission system. IEEE Trans. Commun. Tech. 15(6), 805-811 (1967)

22. P Siohan, C Siclet, N Lacaille, Analysis and design of OFDM/OQAM systems based on filter bank theory. IEEE Trans. Signal Process. 50(5), 1170-1183 (2002)

23. H Bölcskei, Orthogonal frequency division multiplexing based on offset QAM. Adv. Gabor Anal., 321-352 (2003)

24. L Deneire, ed. by M Engels, Beating the wireless channel, in Wireless OFDM Systems, vol. 692 (Springer USA, 2002), pp. 75-93

25. C Lélé, OFDM/OQAM: méthodes d'estimation de canal, et combinaison avec l'accés multiple CDMA ou les systémes multi-antennes. PhD thesis, Conservatoire National des Arts et Métiers, Paris, France, 2008

26. H Lin, P Siohan, P Tanguy, J-P Javaudin, An analysis of the EIC method for OFDM/OQAM systems. J. Commun. 4(1), 52-60 (2009)

27. KEtemad, M-Y Lai, WiMAXTechnology and Network Evolution. (Wiley, IEEE Press, Piscataway, 2011)

28. ETSI, Universal Mobile Telecommunications System (UMTS); Radio Frequency (RF) System Scenarios. ETS TR 125 942, Eur. Telecommun. Standard Institute (2010)

29. M Bellanger, D Le Ruyet, D Roviras, M Terré, J Nossek, L Baltar, Q Bai, D Waldhauser, M Renfors, T Ihalainen, A Viholainen, TH Stitz, J Louveaux, A Ikhlef, V Ringset, H Rustad, M Najar, C Bader, M Payaro, D Katselis, E Kofidis, L Merakos, A Merentitis, N Passas, A Rontogiannis, S Theodoridis, D Triantafyllopoulou, D Tsolkas, D Xenakis, M Tanda, et al., FBMC physical layer: a primer. Technical report, PHYDYAS (Physical layer for dynamic access and cognitive radio)[Online]. Available: http://www.ict-phydyas. org. Accessed Nov 2013

doi:10.1186/1687-6180-2014-54

Cite this article as: Medjahdi et al:: Interference tables: a useful model for interference analysis in asynchronous multicarrier transmission. EURASIP Journal on Advances in Signal Processing 2014 2014:54. 